\title{
Profiles of Cancer Stem Cell Subpopulations in Cholangiocarcinomas
}

Vincenzo Cardinale, ${ }^{\star}$ Anastasia Renzi, ${ }^{\dagger}$ Guido Carpino ${ }^{\ddagger}$ Alessia Torrice, ${ }^{*}$ Maria C. Bragazzi, ${ }^{*}$ Felice Giuliante, Agostino M. De Rose, ${ }^{\S}$ Alice Fraveto, ${ }^{*}$ Paolo Onori, ${ }^{\dagger}$ Chiara Napoletano, ${ }^{\natural}$ Antonio Franchitto, ${ }^{\dagger \|}$ Alfredo Cantafora, ${ }^{*}$ GianLuca Grazi, ** Nicola Caporaso, ${ }^{\dagger \dagger}$ Giuseppe D’Argenio, ${ }^{\dagger \dagger}$ Gianfranco Alpini, ${ }^{\ddagger \ddagger}$ Lola M. Reid, ${ }^{\S \S}$ Eugenio Gaudio, ${ }^{\dagger}$ and Domenico Alvaro*\|

From the Departments of Medico-Surgical Sciences and Biotechnologies, ${ }^{*}$ Anatomical, Histological, Forensic Medicine and Orthopedics Sciences, ${ }^{\dagger}$ and Experimental Medicine, "Sapienza University of Rome, Rome, Italy; the Movement, Human and Health Sciences, ${ }^{\ddagger}$ Division of Health Sciences, University of Rome "Foro Italico," Rome, Italy; the Surgery, Hepatobiliary Unit, ${ }^{\S}$ Catholic University of the Sacred Heart School of Medicine, Rome, Italy; the Eleonora Lorillard Spencer-Cenci Foundation," Rome, Italy; Hepato-Biliary Surgery, ** Regina Elena National Cancer Institute, Rome, Italy; the Gastroenterology Unit $^{\dagger \dagger}$ Department of Clinical and Experimental Medicine, Federico II University of Naples, Naples, Italy; the Central Texas Veterans Health Care System, ${ }^{\ddagger \dagger}$ Scott \& White Digestive Disease Research Center, Department of Medicine, Division Gastroenterology, Scott \& White Healthcare and Texas A\&M System Health Science Center, College of Medicine, Temple, Texas; the Department of Cell Biology and Physiology, ${ }^{\S \S}$ Program in Molecular Biology and Biotechnology, University of North Carolina School of Medicine, Chapel Hill, North Carolina

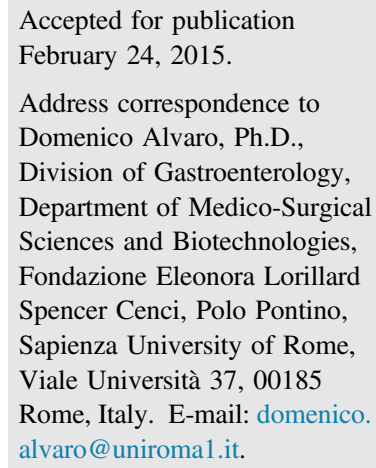

\begin{abstract}
Cholangiocarcinomas (CCAs) comprise a mucin-secreting form, intrahepatic or perihilar, and a mixed form located peripherally. We characterized cancer stem cells (CSCs) in CCA subtypes and evaluated their cancerogenic potential. CSC markers were investigated in 25 human CCAs in primary cultures and established cell lines. Tumorigenic potential was evaluated in vitro or in xenografted mice after s.c. or intrahepatic injection in normal and cirrhotic (carbon tetrachlorideinduced) mice. CSCs comprised more than $30 \%$ of the tumor mass. Although the CSC profile was similar between mucin-intrahepatic and mucin-perihilar subtypes, CD13 ${ }^{+}$CSCs characterized mixed-intrahepatic, whereas $\mathrm{LGR5}^{+}$characterized mucin-CCA subtypes. Many neoplastic cells expressed epithelial-mesenchymal transition markers and coexpressed mesenchymal and epithelial markers. In primary cultures, epithelial-mesenchymal transition markers, mesenchymal markers (vimentin, CD90), and CD13 largely predominated over epithelial markers (CD133, EpCAM, and LGR5). In vitro, CSCs expressing epithelial markers formed a higher number of spheroids than $\mathrm{CD}_{13}{ }^{+}$or $\mathrm{CD}^{+} 0^{+} \mathrm{CSC}$. In s.c. tumor xenografts, tumors dominated by stromal markers were formed primarily by $\mathrm{CD}_{9} 0^{+}$and $\mathrm{CD}_{1} 3^{+}$cells. By contrast, in intrahepatic xenografts in cirrhotic livers, tumors were dominated by epithelial traits reproducing the original human CCAs. In conclusion, CSCs were rich in human CCAs, implicating CCAs as stem cell-based diseases. CSC subpopulations generate different types of cancers depending on the microenvironment. Remarkably, CSCs reproduce the original human CCAs when injected into cirrhotic livers. (Am J Pathol 2015, 185: 1724-1739; http://dx.doi.org/10.1016/j.ajpath.2015.02.010)
\end{abstract}

Supported by an Agenzia Regionale del Lazio per i trapianti e le patologie connesse grant, FIRB grants \#RBAP10Z7FS_001 and RBAP10Z7FS_004, and Federate Athenaeum funds from the Sapienza University of Rome (all to V.C., G.C., E.G., and D.A.); the Dr. Nicholas C. Hightower Centennial Chair of Gastroenterology from Scott \& White Hospital, VA Research Career Scientist Award, VA Merit award, and NIH grants DK58411 and DK062975 (all to G.A.); a sponsored research grant from Vesta Therapeutics (Bethesda, $\mathrm{MD}$ ), a subcontract of a grant to the Hamner Research Institute from the Dow
Chemical Company, NIH grant 1R21CA182322-01, and NIH Cancer Center grant CA016086 (all to L.M.R.); and by resources at the Central Texas Veterans Health Care System.

The views expressed in this article are those of the authors and do not necessarily represent the views of the Department of Veterans Affairs.

V.C. and A.R. contributed equally to this work.

E.G. and D.A. contributed equally to this work as senior authors.

Disclosures: None declared. 
Cholangiocarcinoma (CCA) is the second most common primary hepatic malignancy and arises from the neoplastic transformation of cells in the cholangiocytic lineage. ${ }^{1} \mathrm{CCA}$ is associated with a very bad prognosis with virtually no response to current chemotherapeutics or radiation therapies. ${ }^{1} \mathrm{CCA}$ is classified as intrahepatic (IHCCA), perihilar (pCCA), or distal, characterized by significant differences in terms of epidemiology, pathobiology, and molecular biology. ${ }^{1}$ Recent studies reveal that IHCCA comprises two different forms: mucin-IHCCA constituted by pure mucinsecreting cells and displaying similarities with pCCA, and mixed-IHCCA comprising areas of hepatocytic differentiation and neoplastic ductular reaction. ${ }^{2}$

The cancer stem cell (CSC) hypothesis has been validated recently by the identification of a subpopulation of selfrenewing stem cells that give rise to maturational lineages with a hierarchical organization and are able to divide symmetrically and asymmetrically to generate the tumor mass. ${ }^{3,4}$ CSCs, also referred to as tumor-initiating cells or tumor-propagating cells, are tumorigenic, metastatic, resistant to chemoradio therapies, and responsible for tumor recurrence. ${ }^{3,4}$ For all these reasons, CSCs represent a primary therapeutic target. ${ }^{3,4}$

Recently, several CSC markers have been reported in human CCA, including CD133, ${ }^{5}$ epithelial cell adhesion molecule (EpCAM), ${ }^{6} \mathrm{CD} 44,{ }^{7} \mathrm{CD} 13,{ }^{8}$ and CD90. ${ }^{9}$ In addition, most cells in human CCAs have been demonstrated to coexpress cytokeratin (K)19 and albumin, a feature characterizing hepatobiliary stem/progenitor cells. ${ }^{10}$ Recent reports support further investigations on the role of CSCs in CCA. Unfortunately, very little information exists with respect to CSCs in CCA and its subtypes.

Our aim was to analyze CSCs in different human CCA subtypes, primary cultures obtained from human CCA, and established CCA cell lines.

\section{Material and Methods}

Reagents were purchased from Sigma Chemical (St. Louis, MO) unless otherwise indicated.

\section{Established Human Cell Lines}

Multiple established human cell lines of IH- and pCCA were used: CCLP-1 and HUCCT-1, derived from IHCCA; HuH-28, derived from IHCCA; TFK-1, derived from pCCA; and Mz-ChA1, derived from a gallbladder cancer. CCLP-1 and HUCCT-1 cell lines were obtained from Dr. Anthony J. Demetris (University of Pittsburgh, Pittsburgh, PA). HuH-28 cells were acquired from the Cancer Cell Repository (Tohoku University, Sendai, Japan). TFK-1 cells were kindly provided by Dr. Yoshiyuki Ueno (the Cancer Cell Repository, Tohoku University, Sendai, Japan). MzChA1 cells were a gift from Dr. J. Gregory Fitz (University of Texas Southwestern, Dallas, TX). HuH-28 cells and
Mz-ChA-1 cells were maintained in CMRL 1066 medium containing $10 \%$ fetal bovine serum and $1 \%$ penicillinstreptomycin-glutamine (Gibco/BRL; Life Technologies, Italia srl., Milan, Italy). TFK-1 cells and HUCCT-1 cells were maintained in RPMI 1640 medium containing 10\% fetal bovine serum and $1 \%$ penicillin-streptomycin-glutamine (Gibco/BRL; Life Technologies). CCLPI cells were maintained in RPMI 1640 medium containing 10\% fetal bovine serum and $1 \%$ penicillin-streptomycin-glutamine (Gibco/ BRL; Life Technologies). All human cell lines were maintained at $37^{\circ} \mathrm{C}$ in a humidified atmosphere of $5 \% \mathrm{CO}_{2}$ in air.

\section{Human CCA and Liver Specimens}

Samples of CCA and peritumoral noncancerous liver were obtained from patients submitted to surgical resection, specifically: 17 patients (eight women, aged 50 to 83 years and nine men, aged 57 to 75 years) with IHCCA presenting as a single mass lesion within the liver; eight patients affected by pCCA (Klatskin tumor), (five men, three women, aged 66 to 83 years). Distinctions between IHCCA or pCCA were based on clinical records including surgery reports. ${ }^{1}$

\section{Light Microscopy and IHC in Human CCA Specimens and Tumor Xenografts}

Specimens were fixed in $10 \%$ buffered formalin for 2 to 4 hours, embedded in low-temperature-fusion paraffin $\left(55^{\circ} \mathrm{C}\right.$ to $57^{\circ} \mathrm{C}$ ), and 3- to 4- $\mu \mathrm{m}$ sections were stained with $\mathrm{H} \& \mathrm{E}$, periodic acid-Schiff (PAS) staining system, Sirius Red/Fast Green, according to standard protocols. For immunohistochemistry (IHC), endogenous peroxidase activity was blocked by 30 minutes incubation in methanolic hydrogen peroxide $(2.5 \%)$. Antigens were retrieved by applying Proteinase K (code S3020; Dako, Glostrup, Denmark) for 10 minutes at room temperature in a protocol indicated by the vendor. Sections were then incubated overnight at $4^{\circ} \mathrm{C}$ with primary antibodies (Table 1). Samples were rinsed twice with phosphate-buffered saline (PBS) for 5 minutes, incubated for 20 minutes at room temperature with secondary biotinylated antibody (LSAB + System-HRP, code K0690; Dako), and then incubated with streptavidin-horseradish peroxidase (LSAB+ System-HRP, code K0690; Dako). Diaminobenzidine (Dako) was used as substrate, and sections were counterstained with hematoxylin. For all immunoreactions, negative controls (the primary antibody was replaced with preimmune serum) were also included. Table 1 shows the details of antibodies used in the study.

Sections were examined in a coded fashion by Leica Microsystems DM 4500 B Light and Fluorescence Microscopy (Leica Microsystems, Weltzlar, Germany) equipped with a Jenoptik ProgRes C10 Plus Videocam (Jenoptik, Jena, Germany). Light microscopy and observations were processed with an Image Analysis System (Delta Sistemi, Roma, Italy) and were independently performed by two 
researchers in a blinded fashion (A.R. and G.C.). For IHC staining, the number of positive cells was counted in a random, blinded fashion in six nonoverlapping fields (magnification $\times 20$ ) for each slide and semiquantitatively scored as previously $(0,<1 \% ; 1,1 \%$ to $5 \% ; 2,6 \%$ to $30 \%$; $3,31 \%$ to $50 \%$; and $4=>50 \%) .{ }^{11}$ Necrosis and stromal areas were evaluated, respectively, in hematoxylin and eosin and Sirius Red stains. Necrotic areas were measured by an Image Analysis System (Delta Sistemi), and the total area occupied by necrosis was calculated and expressed as the percentage with respect to the total area. ${ }^{11}$ Sirius Red-stained slides were scanned by a digital scanner (Aperio Scanscope CS System; Aperio Technologies, Oxford, UK) processed by ImageScope. An image analysis algorithm was used to quantify the proportion of picrosiriusstained area. The algorithm was applied to the entire sections of at least three different slides from each specimen. The extent of stromal area was expressed as the proportion $(\%)$ of picrosirius-stained area with respect to the total area.

\section{Cultures of Human CCA Specimens}

CCA samples were subjected to mechanical and enzymatic dissociation. In detail, tumor specimens were transferred into fresh, sterile Dulbeccos' PBS in a 9-cm Petri dish and rinsed. The tissue was transferred to a second dish and minced in a small volume of DMEM high glucose/ DMEM:F12 mixture (1:1, growth medium, Gibco/BRL; Life Technologies) supplemented with $1.8 \times 10^{-4} \mathrm{~mol} / \mathrm{L}$ adenine, $5 \mu \mathrm{g} / \mathrm{mL}$ insulin, $5 \mu \mathrm{g} / \mathrm{mL}$ transferrin, $2 \times 10^{-9}$ $\mathrm{mol} / \mathrm{L}$ triiodothyronine, $1.7 \times 10^{-6} \mathrm{~mol} / \mathrm{L}$ hydrocortisone, $1.0 \times 10^{-6} \mathrm{mg} / \mathrm{L}$ human epidermal growth factor, $5.5 \times$ $10^{-6} \mathrm{mg} / \mathrm{L}$ epinephrine (Sigma-Aldrich, Milan, Italy), $10 \%$ fetal bovine serum (Gibco/BRL; Life Technologies), $100 \mathrm{U} /$ $\mathrm{mL}$ penicillin, and $100 \mu \mathrm{g} / \mathrm{mL}$ streptomycin. The tissue was minced with surgical scalpels into fragments of approximately $1 \mathrm{~mm} .^{3}$ After cutting, the tissue was washed with Dulbeccos' PBS by bench centrifugation $(300 \times g$ for 3 minutes) and resuspended. After washing, fragments were transferred into digestion solution (growth medium with 1 $\mathrm{mg} / \mathrm{mL}$ collagenase type IV, $0.1 \mathrm{mg} / \mathrm{mL}$ hyaluronidase, and $0.1 \mathrm{mg} / \mathrm{mL}$ DNase), and incubated for 12 to 16 hours at $37^{\circ} \mathrm{C}$ in a humidified atmosphere of $5 \% \mathrm{CO}_{2}$ in air.

The effective disaggregation was checked by gently moving the dish; single cells and small clusters were dispersed by gentle pipetting. The cell suspension was filtered with a $100-\mu \mathrm{m}$ cell strainer placed on a $50-\mathrm{mL}$ tube. The cell strainer was washed with $5 \mathrm{~mL}$ of growth medium. The cell suspension was then filtered with a $70-\mu \mathrm{m}$ cell strainer placed on a $50-\mathrm{mL}$ tube. The cell strainer was washed with $5 \mathrm{~mL}$ of growth medium. The cell suspension was then centrifuged at $300 \times g$ for 10 minutes, and the supernatant was discarded. To remove erythrocytes or dead cells, sterile water for injection was used. The cells were resuspended in growth medium and placed into 6-well dish at $37^{\circ} \mathrm{C}$ in a humidified atmosphere of $5 \% \mathrm{CO}_{2}$ in air. When the cells in the dish were expanded, they were transferred into a fresh growth medium supplemented with $10 \%$ fetal bovine serum (Gibco/BRL; Life Technologies), $100 \mathrm{U} / \mathrm{mL}$ penicillin, and $100 \mu \mathrm{g} / \mathrm{mL}$ streptomycin.

For IHC and immunofluorescence (IF), the medium of the CCA cell cultures was removed, and cells were fixed in $4 \%$ paraformaldehyde solution for 10 minutes at room temperature. Cells were rinsed twice with PBS buffer for 2 minutes, blocked, and then incubated 1 hour with the primary antibody at room temperature. After rinsing twice with PBS for 2 minutes, cells were incubated for 40 minutes at room temperature with secondary biotinylated antibody (Vector Laboratories, Milan, Italy), rinsed twice with PBS, and then incubated with Vectastain $\mathrm{ABC}$ reagent (Vector Laboratories) for 20 minutes. Diaminobenzidine (DAB substrate kit; Vector Laboratories) was used as substrate, and sections were counterstained with hematoxylin. Slides were examined by DM 2000 Light and/or Fluorescence Microscopy (Leica Microsystems) equipped with a DFC450 C Videocam (Leica Microsystems). Table 1 shows the details of antibodies used in the study.

\section{Gene Expression Analysis by Real-Time PCR}

Human CCA cell cultures were extracted for total RNA by using TRI Reagent (Sigma-Aldrich, St. Louis, MO) and 1-bromo-3-chloropropane in substitution of chloroform, according to the procedure of Chomczynski and Sacchi. ${ }^{12}$ The isolated RNA was dissolved in $55 \mu \mathrm{L}$ of RNase-free water. RNA quality and quantity was controlled by the Experion Automated Electrophoresis System equipped with the RNA StSens Analysis Chip (Bio-Rad Laboratories, Hercules, CA). The reverse transcription primed by the random hexamer (Invitrogen s.r.l., S. Giuliano Milanese, Italy) was conducted in a $20-\mu \mathrm{L}$ volume with an amount of $2.5 \mu \mathrm{g}$ of total RNA and the M-MLV reverse transcriptase (Invitrogen s.r.l.) according to the manufacturer's directions. Gene expression was determined by real-time PCR with a MX3000P instrument (Agilent, La Jolla, CA) using the averaged cycle threshold $\left(\mathrm{C}_{\mathrm{T}}\right)$ automatically computed by the built-in software from three replicas of each sample. PCR amplifications were conducted into a volume of $25 \mu \mathrm{L}$, with $1.0 \mu \mathrm{L}$ of cDNA template, $12.5 \mu \mathrm{L}$ of $2 \times$ SYBR Green Brilliant QPCR Master Mix (Stratagene, La Jolla, CA), 3 pmoles each of upstream and downstream primer for the gene analyzed, and $0.3 \mu \mathrm{L}$ of diluted reference dye (ROX at a final concentration $30 \mathrm{nmol} / \mathrm{L}$ ). All real-time PCR amplifications were conducted with the cycling program: 10 minutes at $95^{\circ} \mathrm{C}$ followed by 40 cycles $\left(30\right.$ seconds at $95^{\circ} \mathrm{C}$, 30 seconds at $58^{\circ} \mathrm{C}, 30$ seconds at $72^{\circ} \mathrm{C}$ ). The fluorescence detection was performed during the extension step of each cycle. The following genes of interest were measured: CD13 (ANPEP), CD44, CD90 (THY1), CD133 (PROM1), VIM, NANOG, OCT4 (POU5F1), SOX2, SALL4, NIS (SLC5A5, sodium/iodide symporter), E-cadherin ( $\mathrm{CDH1})$, P-cadherin (CDH3), EPCAM, and LGR5. All expression levels were 
Table 1 List of Antibodies Used for IHC and IF

\begin{tabular}{|c|c|c|c|c|}
\hline Name & Host/isotype & Source & Catalog\# & Dilution \\
\hline CD326/EpCAM & Mouse IgG1 & Santa Cruz Biotechnology (Dallas, TX) & sc-59782 & $1: 50$ \\
\hline K7 (cytokeratin 7) & Mouse IgG1 & Dako & M7018 & $1: 100$ \\
\hline K19 (cytokeratin 19) & Mouse IgG1 & Abcam (Cambridge, UK) & ab87014 & $1: 50$ \\
\hline K19 (cytokeratin 19) & Mouse IgG1 & Dako & M0888 & $1: 100$ \\
\hline HepPar-1 & Mouse IgG1 & Dako & M7158 & $1: 50$ \\
\hline CD133/Prominin 1 & Rabbit IgG & Abnova (Taipei, Taiwan) & PAB12663 & $1: 100$ \\
\hline CD13 & Mouse IgG1 & $\begin{array}{l}\text { Novacastra Reagents (Leica Biosystems, } \\
\text { Buffalo Grove, IL) }\end{array}$ & NCL-CD13-304 & $1: 100$ \\
\hline LGR5 & Goat IgG & Santa Cruz Biotechnology & SC-68580 & $1: 50$ \\
\hline Desmin & Mouse IgG1 & Dako & M0760 & $1: 100$ \\
\hline Vimentin & Mouse IgG1 & Santa Cruz Biotechnology & sc-32322 & $1: 100$ \\
\hline Nestin & Mouse IgG1 & Santa Cruz Biotechnology & sc-23927 & $1: 100$ \\
\hline TWIST & Rabbit IgG & Santa Cruz Biotechnology & sc-15393 & $1: 50$ \\
\hline LGR5 & Rabbit IgG & OriGene & TA301323 & $1: 50$ \\
\hline NCAM-PE & Mouse IgG1 & BD Pharmingen (Milan, Italy) & 555.516 & $1: 50$ \\
\hline E-cadherin & Mouse IgG1 & Santa Cruz Biotechnology & sc-21791 & $1: 50$ \\
\hline P-cadherin & Rabbit IgG & Santa Cruz Biotechnology & sc-7893 & $1: 50$ \\
\hline GFAP & Mouse IgG1 & Dako & M0761 & $1: 50$ \\
\hline CD163 & Mouse IgG1 & OriGene & TA506382 & $1: 50$ \\
\hline CD31 & Mouse IgG1 & Dako & M0823 & $1: 50$ \\
\hline CD13 & Mouse IgG1 & Abcam & Ab7417 & $1: 100$ \\
\hline SDF1 & Rabbit IgG & Santa Cruz Biotechnology & sc-28876 & $1: 50$ \\
\hline FAP & Mouse IgG1 & Santa Cruz Biotechnology & sc-65398 & $1: 50$ \\
\hline
\end{tabular}

normalized to the expression of GAPDH housekeeping gene. Table 2 shows the details of primers used in the study (ProbeFinder software version 2.50; Roche, Mannheim, Germany). ${ }^{13-16}$

\section{Flow Cytometry and Magnetic Cell Sorting}

Primary CCA cell cultures were characterized at cell culture passages 2 to 3 and 20 to 30 by using the following antibodies: PE-mouse anti-human CD13 (BD Pharmingen, Milan, Italy), CD90-FITC human, CD133-APC human, CD44-APC human, CD45-PE human, EpCAM-FITC human (Miltenyi Biotec, Milan, Italy), anti-LGR5 mouse monoclonal antibody PE conjugate (OriGene, Unimed Scientifica, Rome, Italy). The fluorescence threshold between negative and positive cells was set on the basis of the reactivity of appropriate nonspecific fluorochrome-conjugated isotypical controls. At least $5 \times 10^{5}$ cells were analyzed using a FACSDiva software version (BD). For magnetic cell sorting, cells were labeled with CD133
(AC133), CD326 (EpCAM), and CD90 MicroBeads, and sorted using the Miltenyi Biotec Cell Isolation Kit, according to the manufacturer's instruction. For magnetic cell sorting of $\mathrm{CD} 3^{+}$and leucine-rich repeat-containing G-protein coupled receptor 5-positive $\left(\mathrm{LGR}^{+}\right)$cells, primary CCA cells were labeled with PE-mouse anti-human CD13 (BD Pharmingen), and anti-Lgr5 mouse monoclonal antibody $\mathrm{PE}$ conjugate (OriGene, Unimed Scientifica). They were then labeled with anti-PE MicroBeads and sorted using the Miltenyi Biotec Cell Isolation Kit, according to the manufacturer's instruction. Isotype-matched mouse immunoglobulins served as controls.

\section{Spheroid Preparation from Primary Human CCA Cultures}

For spheroid formation, primary CCA cell cultures at passages $>20$ were labeled with CD133 (AC133), CD326 (EpCAM), and CD90 MicroBeads, and sorted using the Miltenyi Biotec Cell Isolation Kit, according to the manufacturer's instruction. 
Table 2 Sequences of Primer Pairs (Sense and Antisense, Respectively) Used for Amplifying the Genes of Interest and the Internal Reference Gene (GAPDH) Used for Their Normalization

\begin{tabular}{|c|c|c|c|c|c|}
\hline $\begin{array}{l}\text { Gene } \\
\text { Messenger }\end{array}$ & $\begin{array}{l}\text { Accession } \\
\text { No. }\end{array}$ & Sequences of primer pairs & Length (nt) & Amplicon (bp) & Source \\
\hline \multirow[t]{2}{*}{ GAPDH } & NM_002046 & $5^{\prime}-\mathrm{AGCCACATCGCTCAGACAC}-3^{\prime}$ & 19 & 66 & * \\
\hline & & 5'-GCCCAATACGACCAAATCC-3' & 19 & & \\
\hline \multirow[t]{2}{*}{$C D 13$} & NM_001150 & 5'-CAGTGACACGACGATTCTCC-3' & 20 & 76 & * \\
\hline & & 5'-СCTGTTTCCTCGTTGTCCTT-3' & 20 & & \\
\hline \multirow[t]{2}{*}{$C D 44$} & NM_000610 & 5'-TGCCGCTTTGCAGGTGTAT-3' & 19 & 65 & 13 \\
\hline & & 5'-GGCCTCCGTCCGAGAGA- $3^{\prime}$ & 17 & & \\
\hline \multirow[t]{2}{*}{ CD90 } & NM_006288 & $5^{\prime}-$ AGGACGAGGGCACCTACAC- $3^{\prime}$ & 19 & 107 & * \\
\hline & & $5^{\prime}$-GCCCTCACACTTGACCAGTT-3' & 20 & & \\
\hline \multirow[t]{2}{*}{ CD133 } & NM_002354 & 5'-CCTGGGGCTGCTGTTTATTA-3' & 20 & 161 & * \\
\hline & & $5^{\prime}-\mathrm{ATCACCAACAGGGAGATTGC}-3^{\prime}$ & 20 & & \\
\hline \multirow[t]{2}{*}{ EpCam } & NM_002354 & $5^{\prime}$-ATAACCTGCTCTGAGCGAGTG-3' & 21 & 104 & 14 \\
\hline & & 5'-TGAAGTGCAGTCCGCAAACT-3' & 20 & & \\
\hline \multirow[t]{2}{*}{ VIMENTIN } & NM_003380 & $5^{\prime}-\mathrm{CTGCCAACCGGAACAATGA-3^{ \prime }}$ & 19 & 56 & 14 \\
\hline & & 5'-GTACTCAGTGGACTCCTGCTTT-3' & 22 & & \\
\hline \multirow[t]{2}{*}{ LGR5 } & NM_003667 & 5'-CTTCCAACCTCAGCGTCTTC-3' & 20 & 118 & 14 \\
\hline & & 5'-TTTCCCGCAAGACGTAACTC-3' & 20 & & \\
\hline \multirow[t]{2}{*}{ NANOG } & NM_024865 & $5^{\prime}$-AGATGCCTCACACGGAGACT-3' & 20 & 127 & * \\
\hline & & 5'-TTTGCGACACTCTTCTCTGC-3' & 20 & & \\
\hline \multirow[t]{2}{*}{ OCT4 } & NM_002701 & 5'-TCGAGAACCGAGTGAGAGG-3' & 19 & 125 & * \\
\hline & & 5'-GAACCACACTCGGACCACA-3' & 19 & & \\
\hline \multirow[t]{2}{*}{ SOX2 } & NM_003106 & 5'-TCGAGAACCGAGTGAGAGG-3' & 19 & 85 & * \\
\hline & & 5'-GCAAAGCTCCTACCGTACCA-3' & 20 & & \\
\hline \multirow[t]{2}{*}{ SALL4 } & NM_02,043.3 & $5^{\prime}-$ CGCCCGTGTGTCATGTAGTGAAC-3' & 24 & 123 & 15 \\
\hline & & 5'-TCCGAGAACAGCCGCACTGAGATGGAAG- $3^{\prime}$ & 28 & & \\
\hline \multirow[t]{2}{*}{ NIS } & NM_000453.2 & 5'-TGCGGGACTTTGCAGTACATT-3' & 21 & 133 & 16 \\
\hline & & 5'-TGCAGATAATTCCGGTGGACA-3' & 21 & & \\
\hline \multirow[t]{2}{*}{$\mathrm{CDH} 1$} & NM_004360 & 5'-TCACAGTCACTGACACCAACGA-3' & 22 & 67 & * \\
\hline & & 5'-GGCACCTGACCCTTGTAGTACGT-3' & 20 & & \\
\hline \multirow[t]{2}{*}{$\mathrm{CDH} 3$} & NM_001793.4 & 5'-GGAGGTTTCATCCTCCCTGT-3' & 20 & 88 & * \\
\hline & & 5'-TCCGCTTCTTTCTCACCAAC-3' & 20 & & \\
\hline
\end{tabular}

*Probefinder software, Roche, Mannheim, Germany.

For magnetic cell sorting of $\mathrm{CD} 13^{+}$and LGR5 ${ }^{+}$cells, primary CCA cells were labeled with PE-mouse anti-human CD13 (BD Pharmingen) and anti-Lgr5 mouse monoclonal antibody PE conjugate (OriGene, Unimed Scientifica). They were then labeled with anti-PE MicroBeads and sorted using the Miltenyi Biotec Cell Isolation Kit, according to the manufacturer's instruction. Two thousand sorted cells $\left(\mathrm{CD} 90^{+} / \mathrm{CD} 90^{-}, \mathrm{CD} 13^{+} /\right.$ $\mathrm{CD}_{13}{ }^{-}, \mathrm{CD}_{133^{+} / \mathrm{CD}_{133}-}, \mathrm{LGR}^{+} / \mathrm{LGR}^{-} ; \mathrm{EPCAM}^{+} /$ $\mathrm{EPCAM}^{-}$) were cultured in a serum-free medium of DMEM with high glucose/DMEM:F12 mixture (1:1) (Gibco/BRL; Life Technologies) supplemented with $20 \mathrm{ng} / \mathrm{mL}$ EGF, $10 \mathrm{ng} / \mathrm{mL}$ FGF-2, and 1× B27 (Gibco/BRL; Life Technologies) into each well of 6-well Ultra-Low Attachment plates (Corning, Lowell, MA). After 7 days, spheres were visualized, counted, and sized using light microscopy.

For IF experiments, spheres were incubated with human monoclonal CD326 (EpCAM)-FITC conjugated (1:10; Miltenyi Biotec), human monoclonal CD90-FITC conjugated (1:10; Miltenyi Biotec), human monoclonal CD133/2 (293C3)-PE (1:10; Miltenyi Biotec), Anti-LGR5 mouse monoclonal antibody, clone 2A2, PE conjugated (1:50;
OriGene), anti-human CD13 mouse PE conjugated (1:10; BD Pharmingen), or vimentin-FITC conjugated antibodies (1:50; Santa Cruz Biotechnology, Dallas, TX) for 15 minutes at $4{ }^{\circ} \mathrm{C}$, rinsed with PBS, and then fixed in $4 \%$ paraformaldehyde. Anti-SNAIL rabbit polyclonal (1:50; Santa Cruz Biotechnology), anti-TWIST rabbit polyclonal (1:50; Santa Cruz Biotechnology), anti-P-cadherin (1:50; Santa Cruz Biotechnology) antibodies were incubated for 60 minutes at $4^{\circ} \mathrm{C}$, then the cells were rinsed with PBS and incubated for 40 minutes at room temperature with goat anti-rabbit TRITC (1:400; Abcam, Cambridge, UK) secondary antibody, rinsed with PBS, and fixed in $4 \%$ paraformaldehyde. For all immunoreactions, the cells were counterstained with Vectashield Mounting Medium with DAPI (Vector Laboratories), observed and photographed in a Leica TCS SP2 confocal microscope.

\section{Xenograft Tumorigenicity Experiments}

Spheroids prepared from CSCs immunosorted from human CCA primary cultures were injected (approximately 10,000 


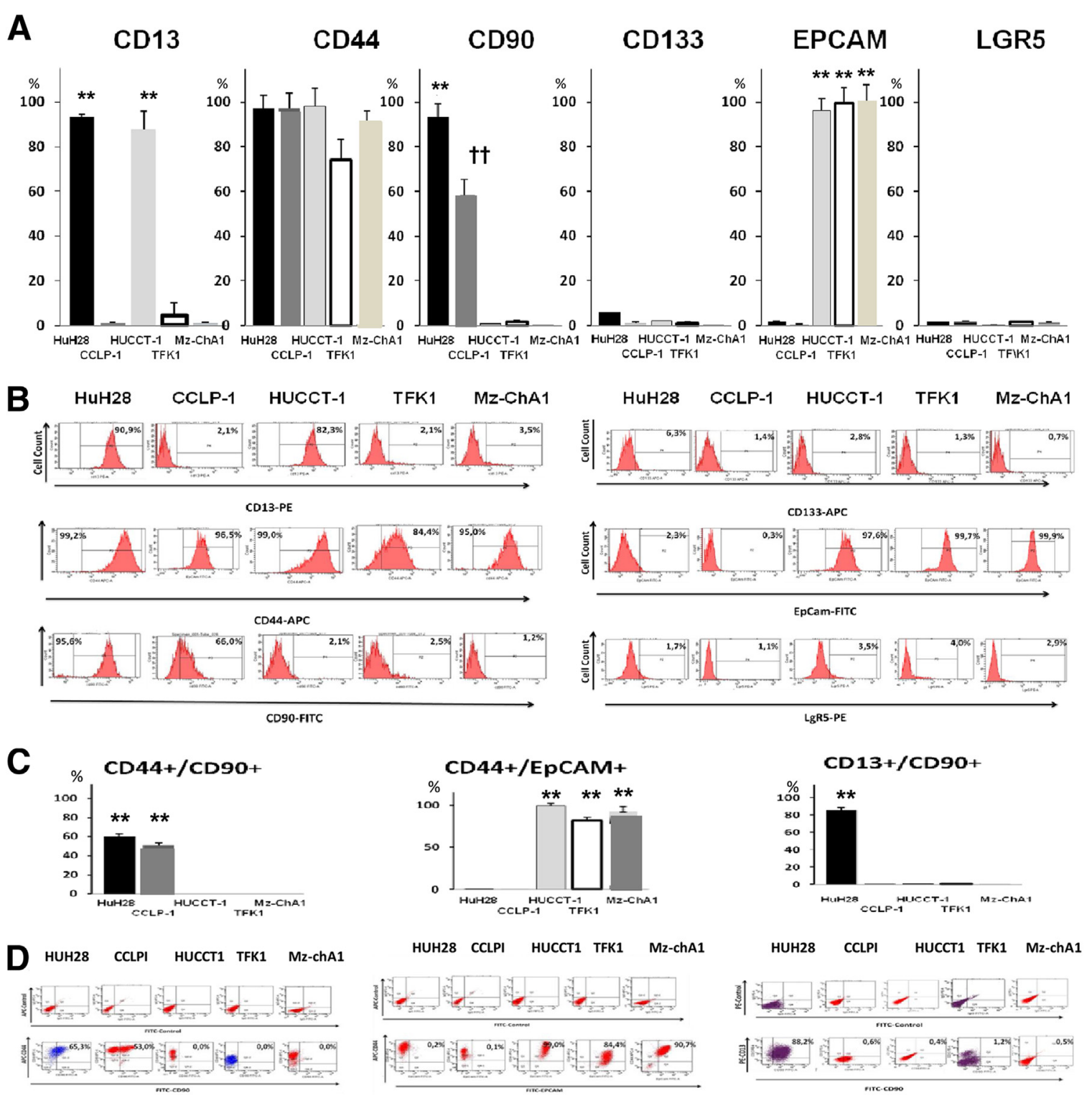

Figure 1 Flow cytometry (FC) analyses of established cell lines of human cholangiocarcinomas (CCAs). A: Bar graphs showing the distribution, as percentages, of cancer stem cell (CSC) subpopulation in established human cancer cell lines as assessed by FC for CSC markers. B: FC representative plots. We investigated: i) HuH-28 and CCLP-I (mucin-negative) and HUCCT-1 (mucin-positive) cell lines derived from human IHCCA; ii) TFK-1 (mucin-positive) cell line derived from pCCA; and iii) Mz-ChA-1 (mucin-positive) cell line derived from gallbladder carcinoma. CD44 expression is high in most of the cell lines ( $>74 \%$ ). $\mathrm{CD} 90^{+}$cells are evident primarily in HuH-28 and CCLP-1. EpCAM positivity characterizes the predominant cell population in the intrahepatic (IHCCA) cell line, HUCCT-1, in perihilar (pCCA) cell lines, TFK-1 and, in gallbladder cancer cell line, Mz-ChA-1. CD133 and LGR5 are minimally expressed. C: Bar graphs showing the distribution, as percentage, of CSC subpopulations coexpressing CSC markers. D: FC representative plots. $\mathrm{CD} 44^{+} / \mathrm{CD} 90^{+}$cells predominate in HuH-28 and CCLP-1 cell lines. CD44 ${ }^{+} / \mathrm{EpCAM}^{+}$cells are prevalent in HUCCT-1, TFK-1, and Mz-ChA-1 cell lines. $\mathrm{CD} 13^{+} / \mathrm{CD}^{+} 0^{+}$cells predominate only in HuH-28 cells. ${ }^{* *} P<0.01$ versus the other cell lines; ${ }^{\dagger \dagger} P<0.01$ versus HuH28.

cells) s.c. or in the liver of normal or cirrhotic (carbon tetrachloride-induced) mice.

\section{S.C. Implantation}

Male NOD/SCID mice, 4 to $6 \mathrm{wk}$ old, purchased from Charles River Laboratories (Wilmington, MA) were maintained under standard conditions and cared according to the institutional guidelines for animal care. Spheroids prepared from CSCs immunosorted from human CCA primary cultures were injected (approximately 10,000 cells) s.c. into the right and left mid-abdominal areas. Tumor xenograft formation was followed by macroscopic inspection, and after 2 to 4 months, mice were killed by cervical dislocation; tumors were then removed and fixed in $10 \%$ neutral buffered formalin for histology and IHC.

Intrahepatic Injection

Spheroids prepared from CSCs immunosorted from human CCA primary cultures of mucin-IHCCA or mixed-IHCCA 


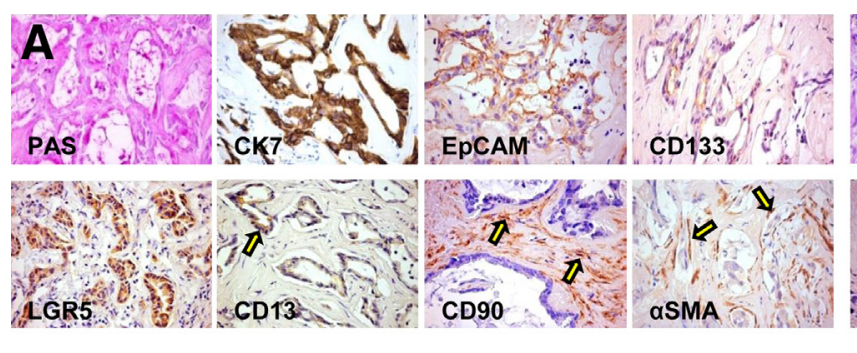

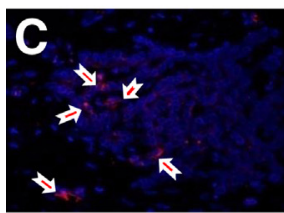

Nuclei Desmin

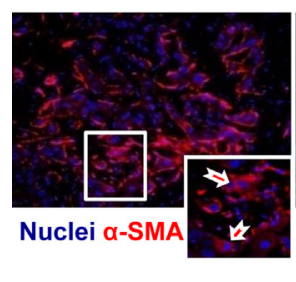

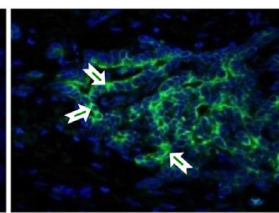

Nuclei CD133

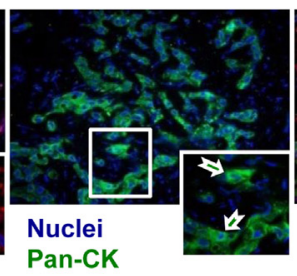

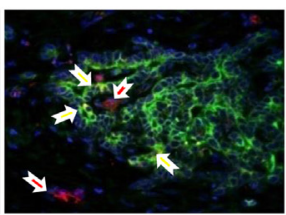

Nuclei Desmin CD133

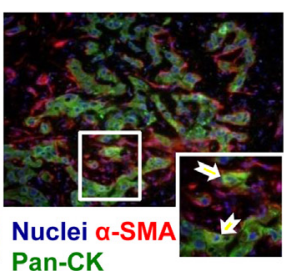

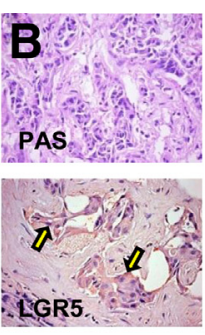
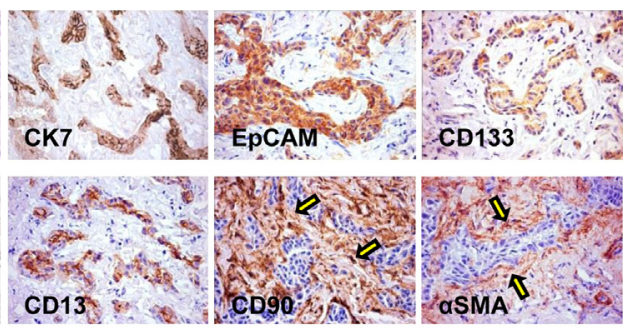

CD133

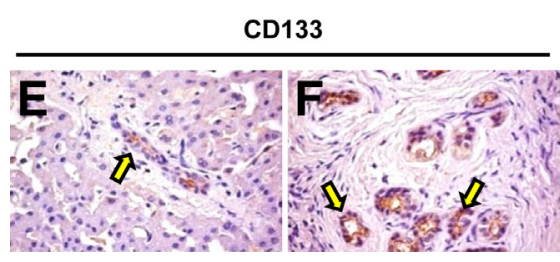

CD90
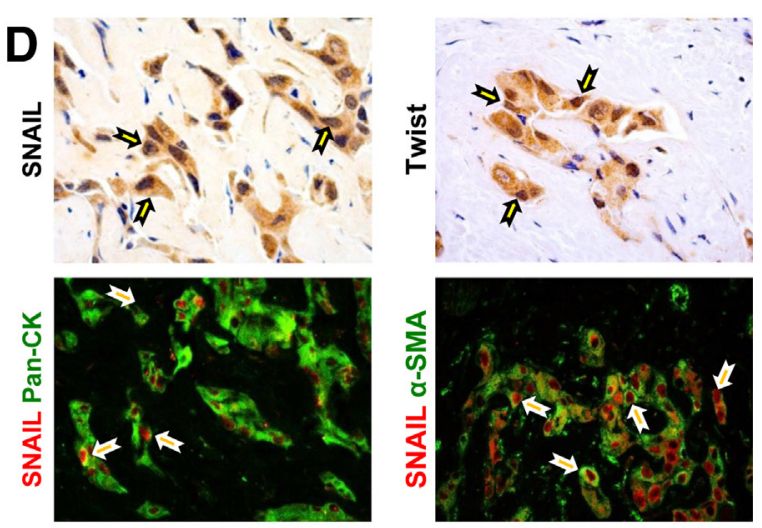

CD13
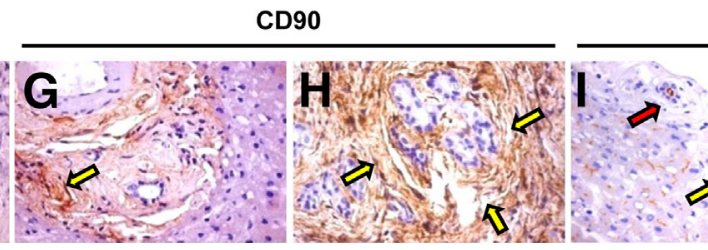

Figure 2 Immunophenotypic traits of human mucin-intrahepatic (IHCCA) and mixed-IHCCA. A: Periodic-acid Schiff (PAS) stain and immunohistochemistry (IHC) for keratin (K)7, EpCAM, LGR5, CD133, CD13, CD90, and $\alpha$-smooth muscle actin ( $\alpha$-SMA) on human mucin-IHCCAs. Mucin-IHCCAs are diffusely positive for PAS, K7, EpCAM, LGR5, and CD133. By contrast, CD13 is restricted to a small subpopulation of tumor epithelial cells (arrow). CD90 and $\alpha$-SMA are primarily expressed by tumor stromal cells (arrows). B: PAS stain and IHC for K7, EpCAM, LGR5, CD133, CD13, CD90, and $\alpha$-SMA on human mixed-IHCCAs. Mixed-IHCCAs are diffusely positive for K7, EpCAM, CD13, and CD133. By contrast, LGR5 is restricted to a small subpopulation of tumor epithelial cells (arrows). CD90 and $\alpha$-SMA were primarily expressed by tumor stromal cells (arrows). C: Double immunofluorescence (IF) for Desmin (red) and CD133 (green) in mucin-IHCCA; double IF for $\alpha$-SMA (red) and pan-cytokeratin (Pan-CK; green) in mixed-IHCCAs. Approximately $10 \%$ of tumor epithelial cells (CD133 ${ }^{+}$or Pan-CK ${ }^{+}$, in green) express mesenchymal markers (desmin or $\alpha$-SMA, in red) both in mucin- and in mixed-IHCCA. Yellow arrows indicate cells coexpressing epithelial and mesenchymal markers (yellow cells in merged images). Nuclei are shown in blue. Separate channels are shown. Boxed areas are shown magnified in the insets (bottom panels). D: Upper panels: IHC for SNAIL and Twist in human CCA specimens shows the expression of the two antigens within the nuclei of epithelial CCA cells (arrows). Lower panels: Double IF for SNAIL (red) and Pan-CK (green) in human CCA specimens confirms the expression of SNAIL within nuclei of epithelial CCA cells (arrows). Double IF for SNAIL (red) and $\alpha$-SMA (green) in human CCA specimens shows the presence of CCA cells expressing both $\alpha$-SMA and SNAIL. Images from mixed-IHCCA samples are displayed. E-J: IHC for CD133, CD13, and CD90 on human nontumor tissue surrounding CCA specimens. CD133 expression (E and $\mathbf{F})$ is restricted to a few cells within Canals of Hering $(\mathrm{CoH})$ and bile ductules $(\mathbf{E}$, arrow) and to peribiliary glands (PBGs) within larger bile ducts ( $\mathbf{F}$, arrows). CD90 ( $\mathbf{G}$ and $\mathbf{H})$ is mostly expressed by stromal cells in the portal spaces ( $\mathbf{G}$, arrow) and around PBGs (H, arrows). CD13 (I and $\mathbf{J})$ is diffusely expressed by hepatocytes, cells within $\mathrm{CoH} /$ bile ductules (I, yellow arrows), cholangiocytes of interlobular bile ducts (I, red arrow), and PBGs (J, arrow). Original magnification: $\times 20($ A - C, D, lower panels) $\times 40($ D, upper panel).

were injected (approximately 10,000 cells) directly into the livers of normal and cirrhotic 13-wk-old male NOD/SCID mice. Spheroids were suspended in $100 \mu \mathrm{L}$ of vehicle [50\% hyaluronic acid gel (Sigma-Aldrich, St. Louis, MO) and prepared in Kubota's medium] and injected into the frontal lobe. Liver cirrhosis was induced by intraperitoneal injections, twice a week for 7 weeks with carbon tetrachloride, $0.2 \mathrm{~mL} / 100 \mathrm{~g}$ in refined olive oil (1:1), according to a wellestablished model. ${ }^{17}$ As controls, the olive oil vehicle was injected in control mice and also in a distant liver lobe of the mice. At 4 weeks after the injection, animals were sacrificed in a box with anesthesia under carbon dioxide atmosphere, and their livers were harvested. Injected lobes were fixed in formalin for histological and IHC analyses.

Intrahepatic xenografts were also performed in immunocompetent cirrhotic BALB/c male mice (13 week old; Harlan Laboratories, Correzzana, Italy). CD13 ${ }^{+}$, CD133 ${ }^{+}$, $\mathrm{CD} 90^{+}$spheroids (approximately 10,000 cells), suspended in $100 \mu \mathrm{L}$ of vehicle [50\% hyaluronic acid gel (SigmaAldrich) in Kubota's medium] were injected in the frontal lobe, and for 28 days after the cell injection, animals were pharmacologically immunosuppressed through continuous infusion of cyclosporine $(1 \mathrm{mg} / \mathrm{kg}$ per day) by osmotic micropumps (Alzet model 1004 pump; Durect Corporation, 

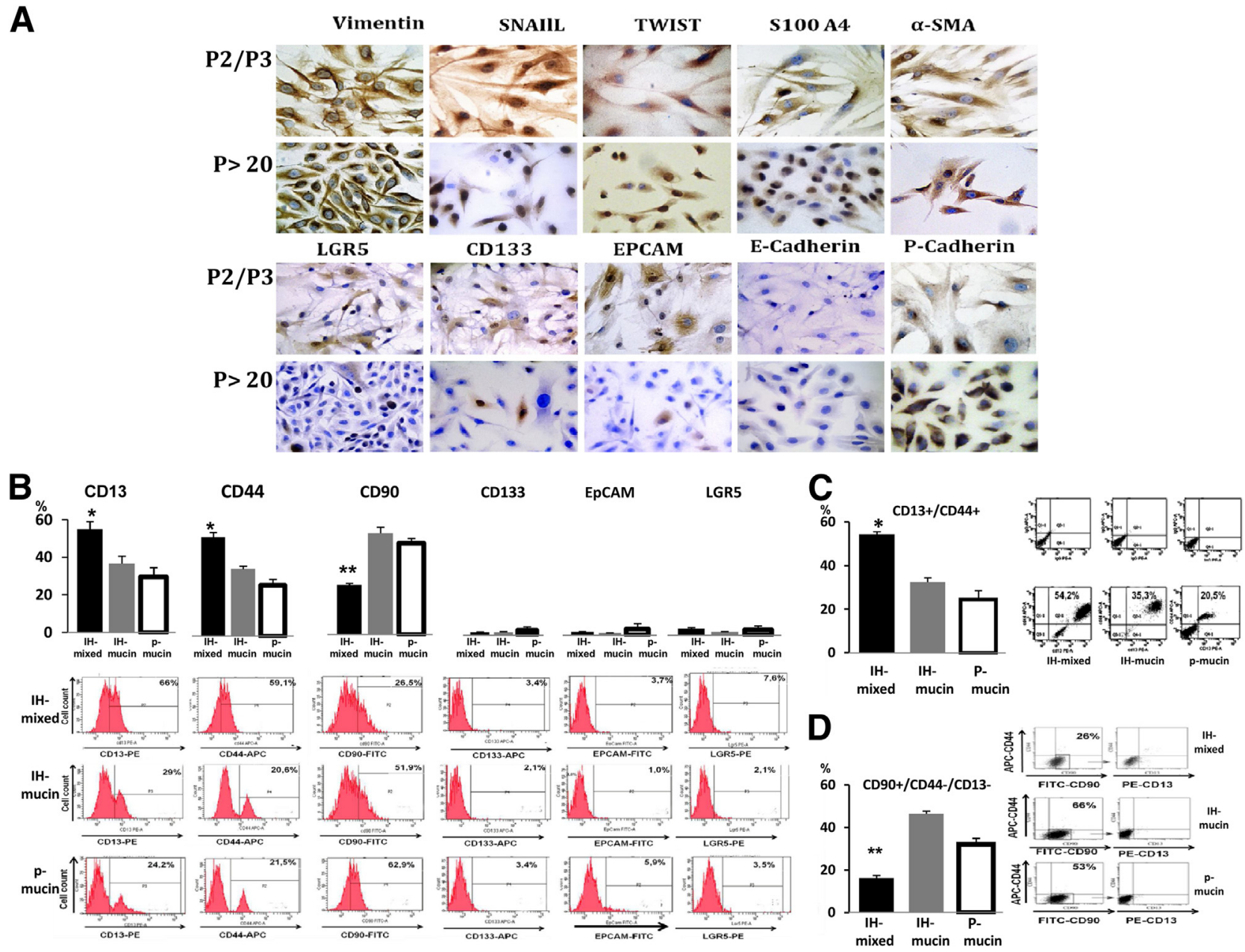

Figure 3 Human cholangiocarcinoma (CCA) primary cultures. A: Immunohistochemical analyses of CCA primary cultures after 2 to 3 passages (P2/P3) and 20 to 30 passages $(P>20)$. The expression of mesenchymal and epithelial-mesenchymal transition markers (vimentin, $\alpha$-SMA, SNAIL, Twist, S100A4, Pcadherin) largely predominate over that of epithelial markers (CD133, EpCAM, LGR5, E-cadherin) in all passages examined. During progression of primary cultures ( $P>20$ versus P2/P3) SNAIL- and Twist-positive cells tend to increase, whereas EpCAM-, LGR5-, and CD133-positive cells tend to decrease. Similar diffuse positivity for vimentin, $\alpha$-SMA, and S100A4 is observed in all passages. B-D: Flow cytometric (FC) analyses of primary cultures (20 to 30 passages) obtained from mixed-IHCCA (IH-mixed), mucin-IHCCA (IH-mucin), and mucin-pCCA (p-mucin) subtypes; representative FC plots. B: Cells positive for CD13, CD44, and CD90 largely predominate with respect to CD133, EpCAM, and LGR5. CD13 ${ }^{+}$and CD44 ${ }^{+}$cells predominate in mixed-IHCCA with respect to mucinIHCCA or mucin-pCCA, whereas the opposite is found for CD90 cells. C: Cells double-positive for CD13 and CD44 (CD13 $\left.{ }^{+} / \mathrm{CD}_{4} 4^{+}\right)$predominate in mixed-IHCCA

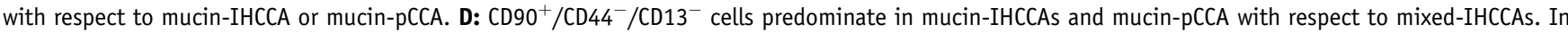
these experiments, $\mathrm{CD}^{+}$cells are gated and further analyzed for the expression of $\mathrm{CD} 44$ and $\mathrm{CD} 13$ markers. ${ }^{*} P<0.05$, ${ }^{* *} P<0.01$ versus mucin-CCAs. Original magnification: $\times 40($ A).

Cupertino, CA) inserted into the peritoneum at the moment of the cell injection. Liver cirrhosis was induced as described for NOD/SCID mice. As controls, the cell vehicle was injected in control mice and also in a distant liver lobe. Four weeks after the injection, animals were sacrificed in a box for anesthesia under carbon dioxide atmosphere and their livers were harvested. Injected lobes were fixed in formalin for histological and IHC analyses.

\section{Statistical Analysis}

Data are presented as the arithmetic means \pm SD or SEM, as indicated. Statistical analyses were conducted using the paired or unpaired Student's $t$-test as appropriate or the analysis of the variance when multiple comparisons were performed. $P<0.05$ was considered statistically significant.

\section{Results}

CSC Marker Expression Profiles in Established CCA Cell Lines

We investigated a number of CSC markers, suggested by recent literature, ${ }^{5-9}$ in different established cell lines derived from: i) human IHCCA: HuH-28 and CCLP-I, mucin-negative (Pas); ii) HUCCT-1, mucin-positive; 
human pCCA: TFK-1, mucin-positive; and iii) human gallbladder cancer: Mz-ChA-1, mucin-positive.

By flow cytometry (FC) (Figure 1, A and B), CD44 ${ }^{+}$was highly represented in all cell lines. $\mathrm{CD} 90^{+}$was expressed primarily by HuH-28 and CCLP-1 cell lines, whereas it was negligible in HUCCT-1, TFK-1, and Mz-ChA-1 cell lines. By contrast, the epithelial stem cell marker, EpCAM, was dominant in HUCCT-1, TFK-1, and Mz-ChA-1 cell lines. This marker was scarcely evident in HuH-28 and CCLP-1 cell lines. The other epithelial markers, CD133 and LGR5, were minimally expressed (Figure 1, A and B), and the hematopoietic cell marker CD45 was not found at all in all examined cell lines.

When coexpression of markers was evaluated (Figure 1, C and $\mathrm{D}), \mathrm{CD} 44^{+} / \mathrm{CD} 90^{+}$cells predominated in $\mathrm{HuH}-28$ and CCLP-1 cells; this cell population expressed vimentin with real-time PCR analyses (data not shown). By contrast, $\mathrm{CD} 44^{+} / \mathrm{EpCAM}^{+}$cells were prevalent in HUCCT-1, TFK-1, or Mz-ChA-1 cell lines; these cells were negative for vimentin expression by real-time PCR (data not shown). Finally, $\mathrm{CD} 13^{+} / \mathrm{CD} 90^{+}$cells (vimentin positive by real-time PCR) were the predominant cell population $(85 \%)$ only in HuH-28 cells.

By real-time PCR (Supplemental Figure S1), vimentin was significantly expressed only by $\mathrm{HuH} 28$ and CCLP1 cell lines. CD44 mRNA was expressed at almost similar levels in all established cell lines. In agreement with FC, mRNAs for CD90 were expressed almost exclusively in $\mathrm{HuH} 28$ and CCLP1 cells, whereas the epithelial marker EpCAM was found only in HUCCT-1, TFK-1, and MzChA-1 cell lines. CD13 was highly expressed only in HuH-28 cells. CD133 and LGR5 were minimally expressed in all cell lines.

\section{Pathology of Human CCA Specimens and IHC Expression of CSC Surface Markers}

By morphology and PAS staining, according to Komuta et al, ${ }^{2}$ the CCAs were classified as: i) mixed-IHCCA $(n=9)$ (Figure 2), displaying histological diversity such as focal hepatocytic differentiation, neoplastic ductular reaction, and focal mucin producing areas; ii) mucin-IHCCA $(n=8)$ (Figure 2), mostly composed of mucin-producing cells; and iii) mucin-pCCA ( $n=8$, Klatskin tumor), mostly composed of mucin-producing cells.

In CCA specimens, the areas occupied by stromal components slightly predominated over those with epithelia (Figure 2, $\mathrm{A}$ and B) $[\alpha$-smooth muscle actin ( $\alpha$-SMA)] and without distinctions between mixed-IHCCAs $(58.1 \pm 7.2 \%)$ and pure mucin-CCAs (55.4 $\pm 9.6 \%$ ), the latter being similar regardless of an intrahepatic or perihilar location. PAS positivity was diffuse in the epithelial neoplastic areas of only mucinIHCCAs (Figure 2A) and pCCAs. By IHC, in all specimens, diffuse positivity for K7, EpCAM, and CD133 was found in epithelial neoplastic areas, without distinctions between mixed-IHCCAs and mucin-IHCCAs (Figure 2, A and B).
Table 3 Additional Markers Investigated by IHC or IF in Primary Cultures ( $>20$ Passages) of Mucin- or Mixed-IHCCA

\begin{tabular}{lll}
\hline Markers (technique) & Mucin-IHCCA & Mixed-IHCCA \\
\hline E-cadherin (IHC-IF) & $0 \%$ & $0 \%$ \\
CD31 (IHC-IF) & $0 \%$ & $0 \%$ \\
GFAP (IHC) & $0 \%$ & $0 \%$ \\
CD163 (IHC) & $0 \%$ & $0 \%$ \\
SDF1 (IF) & $0 \%$ & $0 \%$ \\
FAP (IF) & $0 \%$ & $0 \%$ \\
Periostin (IF) & $18 \pm 3.0 \%$ & $14 \pm 3.5 \%$ \\
$\alpha$-SMA (IHC-IF) & $20 \pm 3.6 \%$ & $15 \pm 4.3 \%$ \\
S100A4 (IHC-IF) & $100 \%$ & $100 \%$ \\
SNAIL (IHC-IF) & $88 \pm 3.2 \%$ & $86 \pm 3.5 \%$ \\
TWIST (IHC-IF) & $86 \pm 1.5 \%$ & $85 \pm 2.5 \%$ \\
NCAM (IF) & $98 \pm 2.0 \%$ & $96 \pm 3.0 \%$ \\
CK19 (IF) & $13 \pm 3.0 \%$ & $12 \pm 2.7 \%$ \\
Vimentin (IHC-IF) & $100 \%$ & $100 \%$ \\
Nestin (IF) & $100 \%$ & $100 \%$ \\
\hline
\end{tabular}

Data indicate percentage of cells positive and are expressed as means \pm SD.

$\alpha$-SMA, $\alpha$-smooth muscle actin; CK, cytokeratins; FAP, fibroblast-activation protein; GFAP, glial fibrillary acidic protein; IF, immunofluorescence; IHC, immunohistochemistry; IHCCA, intrahepatic cholangiocarcinoma; NCMA, neural cell adhesion molecule; SDF1, stromal-derived factor.

LGR5 was diffusely expressed in mucin-CCA (both IHCCA and pCCA) but with few tumor epithelial cells in mixedIHCCAs (semiquantitative score $=2.2 \pm 0.8$ versus $0.8 \pm 0.7, P<0.05$ ) (Figure 2, A and B). By contrast, CD13 positivity, considered as a marker of quiescent $\mathrm{CSCs},{ }^{3,4}$ was diffuse in mixed-IHCCA and rare in mucin-CCAs (semiquantitative score $=2.4 \pm 1.1$ versus $0.9 \pm 0.8, P<0.05$ ) (Figure 2, A and B). Moreover, in all CCA samples, CD90 (and also vimentin, data not shown) expression was mostly restricted to stromal cells within the tumors (Figure 2, A and B). No morphological or IHC differences were found between mucin-IHCCAs and mucin-pCCAs.

Double IF analyses demonstrated that approximately $10 \%$ of the tumor epithelial cells [Pan-CK ${ }^{+}$(ie, biliary keratins K7 and $\mathrm{K} 19)$ or $\mathrm{CD} 133^{+}$] also co-express mesenchymal markers (vimentin or $\alpha$-SMA) (Figure 2C). In addition, approximately $30 \%$ of tumor epithelial cells were positive for both SNAIL and Twist, markers of epithelial-mesenchymal transition (EMT) (Figure 2D).

In nontumoral surrounding tissue, the expression of CD133 (Figure 2, E and F) and LGR5 was restricted to peribiliary glands (PBGs) within larger bile ducts and in a few cells within the canals of Hering and bile ductules. CD90 (Figure 2, G and $\mathrm{H}$ ) and $\boldsymbol{\alpha}$-SMA were mostly restricted to stromal cells. CD13 was diffusely expressed by hepatocytes, by cells within canals of Hering and bile ductules, cholangiocytes of interlobular bile ducts, and PBGs (Figure 2, I and J).

By Western blot analysis of human CCA samples, E-cadherin expression was markedly lower $(P<0.01)$ with respect to noncancerous peritumoral area, whereas the 
A
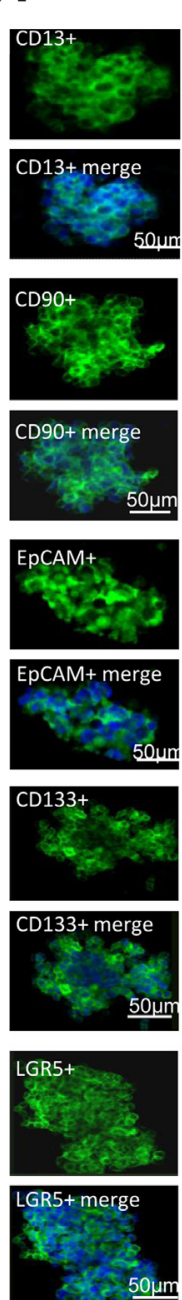
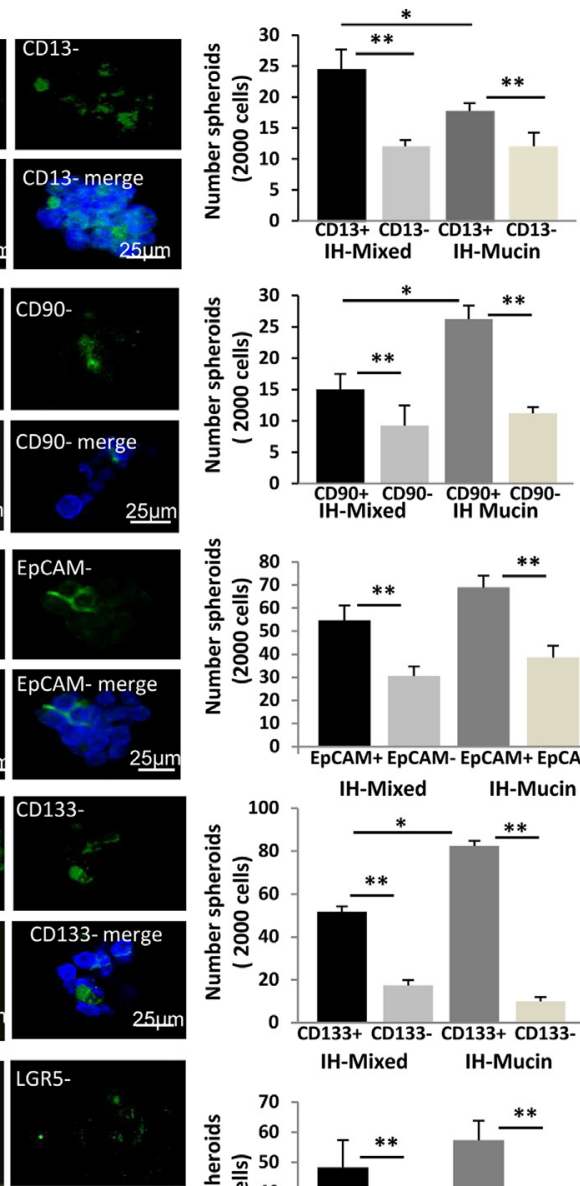

LGR5- merge
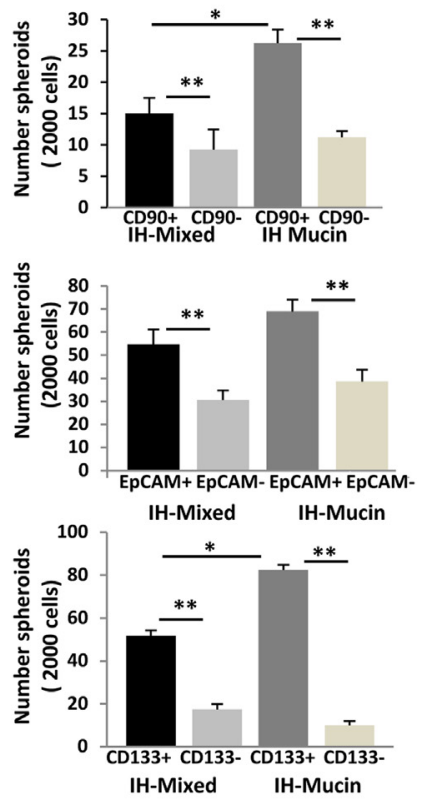

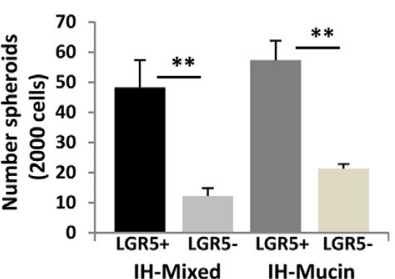

B
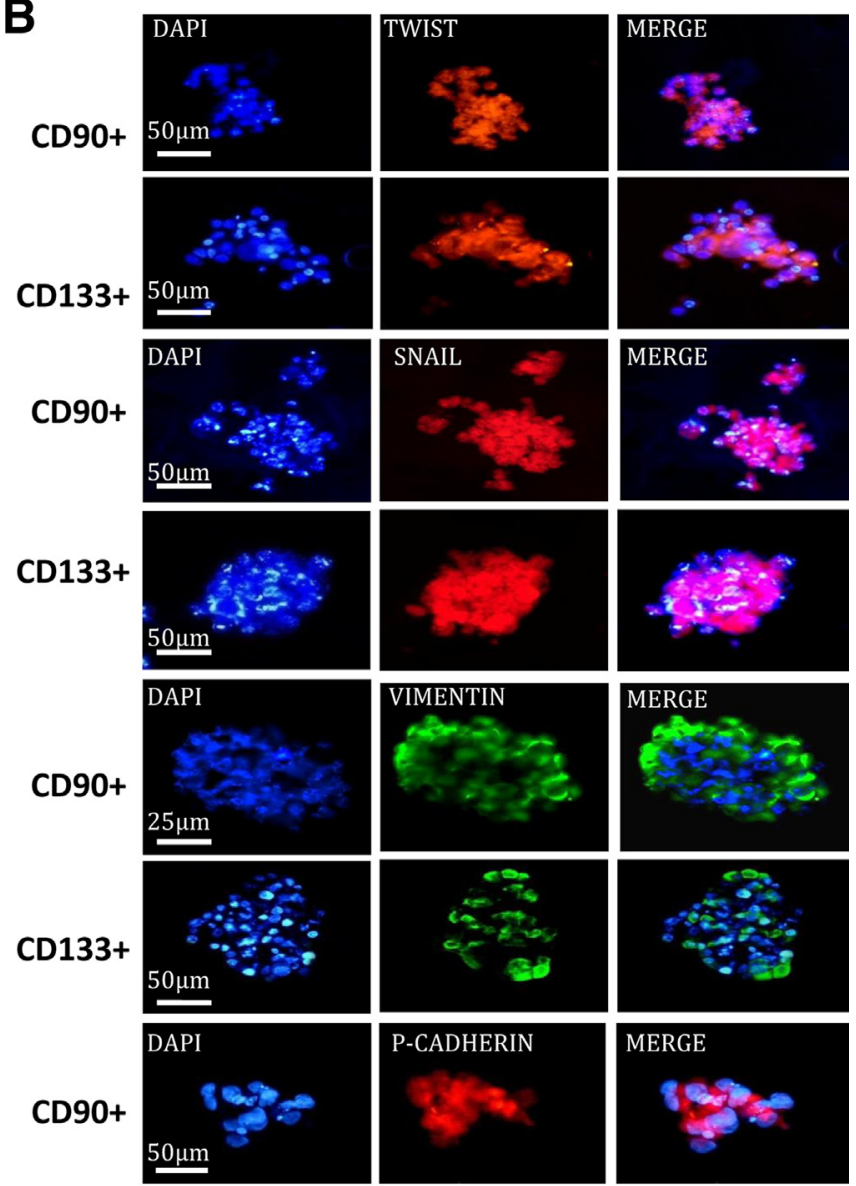

CD133+
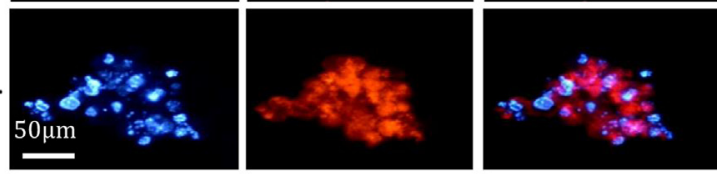

Figure 4 Spheroids formed by cholangiocarcinomas (CCAs) subpopulations. A: We determined the capacity of spheroid formation by cancer stem cell (CSC) subpopulations immunosorted from CCA primary cultures; the number of spheroids (see bar graph) formed in vitro being a self-renewal index. By immunofluorescence (IF) for the marker used for immunosorting, we tested the purity of the formed spheroids. Spheroids formed by $\mathrm{CD}^{+} 0^{+}$cells are enriched for $\mathrm{CD} 0^{+}$cells, whereas the negative counterpart $\mathrm{CD} 90^{-}$forms spheroids where $\mathrm{CD} 90^{+}$cells are $<5 \%$, indicating that these spheroids are formed by other $\mathrm{CSC}$ subpopulations. Each subpopulation forms spheroids efficiently, reaching a size of 100 to $500 \mu \mathrm{m}$ after 7 days in culture. IF (nuclei were stained with DAPI) shows the enrichment in the spheroids by the immunosorted cell subpopulation. CD133, EpCAM, and LGR5 form a higher $(P<0.01)$ number of spheroids compared to cells expressing the mesenchymal cell marker, CD90, or CD13 (note that scales are different). CD13 ${ }^{+}$cells from mixed-IHCCA (IH-mixed) form a higher number of spheroids than $\mathrm{CD}_{13}{ }^{+}$cells immunoselected from mucin-IHCCA (IH-mucin), whereas the opposite is observed for $\mathrm{CD}^{+} 0^{+}$or $\mathrm{CD} 133^{+}$cells (mucin > mixed.) B: Spheroids formed by $\mathrm{CD}_{133^{+}}$and $\mathrm{CD}^{+} 0^{+} \mathrm{CSC}$ subpopulations were analyzed by IF for markers of epithelial-mesenchymal transition (Twist, SNAIL, vimentin, $\mathrm{P}$-cadherin). Spheroids show positive staining for Twist, SNAIL, vimentin, and P-cadherin without differences between $\mathrm{CD}_{133^{+}}$and $\mathrm{CD} 90^{+}$ spheroids. ${ }^{*} P<0.05,{ }^{*} P<0.01$. Original magnification: $\times 30(\mathbf{A}) ; \times 40(\mathbf{B})$.

opposite was found for vimentin expression that predominated in cancerous tissues $(P<0.05)$ (Supplemental Figure S2).

\section{Expression of CSC Markers in Primary Cultures of Human CCA Specimens}

Cells isolated from human CCA specimens did not survive in serum-free Kubota's medium, a medium lacking growth factors, and tailored for normal endodermal stem/progenitor cells. ${ }^{18}$ This suggests that CSCs in CCAs are biologically distinct from normal stem/progenitor cells of liver, biliary tree, and pancreas. Instead, successful culturing was obtained when cells were plated in media supplemented with different growth factors. Cultures showed a steady growth rate from the third passage and could be grown to more than 40 passages. Before culturing, the distribution of CSC markers (IF) largely corresponds to IHC performed in situ in CCAs. After culturing, even after two to three passages, the expression of mesenchymal and EMT markers (vimentin, $\alpha$-SMA, SNAIL, S100A4, Twist, P-cadherin) largely predominated over that of epithelial markers (CD133, EpCAM, 
Table 4 Tumor Xenografts Obtained 2 Months after S.C. Injection of Spheroids Prepared from Different Subpopulations of Immunosorted CSCs

\begin{tabular}{|c|c|c|c|}
\hline $\begin{array}{l}\text { IHCCA } \\
\text { subtypes }\end{array}$ & $\begin{array}{l}\text { CSC } \\
\text { subpopulation }\end{array}$ & $\begin{array}{l}\text { No. Mice } \\
\text { with tumor/No. } \\
\text { injected mice }\end{array}$ & $\begin{array}{l}\text { Tumor volume } \\
\left(\mathrm{mm}^{3} \pm \mathrm{SD}\right)\end{array}$ \\
\hline \multicolumn{4}{|c|}{ Mucin-IHCCA } \\
\hline & $\mathrm{CD}_{13}{ }^{+}$ & $3 / 3^{*}$ & $1500 \pm 76^{*}$ \\
\hline & $\mathrm{CD}_{13}{ }^{-}$ & $3 / 3$ & $650 \pm 78$ \\
\hline & $\mathrm{CD}^{\circ} 0^{+}$ & $3 / 3^{*}$ & $2200 \pm 100^{*}$ \\
\hline & $\mathrm{CD}^{-} 0^{-}$ & $3 / 3$ & $450 \pm 130$ \\
\hline & $\mathrm{CD}_{133^{+}}$ & $3 / 6$ & $500 \pm 55$ \\
\hline & CD133- & $3 / 6$ & $285 \pm 75$ \\
\hline & $\mathrm{EpCAM}^{+}$ & $0 / 5^{\dagger}$ & \\
\hline & EpCAM $^{-}$ & $0 / 5^{\dagger}$ & \\
\hline & $\mathrm{LGR5}^{+}$ & $0 / 5^{\dagger}$ & \\
\hline & $\mathrm{LGR5}^{-}$ & $0 / 5^{\dagger}$ & \\
\hline \multicolumn{4}{|c|}{ Mixed-IHCCA } \\
\hline & $\mathrm{CD}_{13}{ }^{+}$ & $5 / 6^{*}$ & $1400 \pm 848^{*}$ \\
\hline & $\mathrm{CD}_{13}{ }^{-}$ & $3 / 6$ & $650 \pm 350$ \\
\hline & $\mathrm{CD}^{\circ} 0^{+}$ & $5 / 6^{*}$ & $1300 \pm 141^{*}$ \\
\hline & $\mathrm{CD}^{-} 0^{-}$ & $3 / 6$ & $450 \pm 185$ \\
\hline & $\mathrm{CD}_{133^{+}}$ & $3 / 7$ & $400 \pm 121$ \\
\hline & CD133- & $3 / 7$ & $220 \pm 120$ \\
\hline & $\mathrm{EpCAM}^{+}$ & $0 / 5^{\dagger}$ & \\
\hline & $\mathrm{EpCAM}^{-}$ & $0 / 5^{\dagger}$ & \\
\hline & $\mathrm{LGR5}^{+}$ & $0 / 5^{\dagger}$ & \\
\hline & $\mathrm{LGR5}^{-}$ & $0 / 5^{\dagger}$ & \\
\hline
\end{tabular}

Spheroids obtained from CSCs immunosorted from primary cultures of human mucin- or mixed-IHCCA were injected s.c. (approximately 10,000 cells) in male NOD/SCID mice and tumor xenografts evaluated 2 months later.

${ }^{*} P<0.05$ versus $\mathrm{CD} 133^{+}$

${ }^{\dagger} \mathrm{EpCAM}^{+}$or $\mathrm{Lgr5}^{+}$spheroids formed tumors only after 3 to 4 months; in all cases, marker-positive spheroids formed tumors of larger volume than marker-negative spheroids.

CSC, cancer stem cell; IHCCA, intrahepatic cholangiocarcinoma.

LGR5) (Figure 3A). During progression of the primary cultures ( 2 to 3 passages versus 20 to 30 passages) (Figure 3A), cells positive for SNAIL and Twist increased, whereas EpCAM, LGR5, and CD133 decreased. Diffuse positivity for vimentin, $\alpha$-SMA, and S100A4 was found in all passages without differences between passages 2 to 3 and 20 to 30 (Figure 3A).

Primary cultures ( $>20$ passages) were investigated for CSC profile and used for spheroid preparation (see S.C. Tumor Xenografts or Intrahepatic Tumor Xenografts). In these cultures, cells were (Table 3) negative for E-cadherin, for markers of tumor-associated macrophages (CD163), activated hepatic stellate cells (GFAP), ${ }^{19,20}$ endothelial cells (CD31), ${ }^{19,20}$ fibroblast-activation protein (FAP),${ }^{19,20}$ stromal-derived factor (SDF1), ${ }^{19,20}$ whereas a weak positivity for periostin (a fibroblast-secreted extracellular matrix protein) $)^{19,20}$ was observed in $<20 \%$ cells and only in $\alpha$-SMA ${ }^{+}$cells. Nestin, a stem cell marker, ${ }^{19,20}$ labels $100 \%$ of the cells.

In primary cultures, FC (20 to 30 passages) demonstrated that cells positive for $\mathrm{CD} 13, \mathrm{CD} 44$, and CD90 largely predominated as compared to CD133, EpCAM, and LGR5
(25\% to $55 \%$ versus $1 \%$ to $7 \%, P<0.01$ ) (Figure $3 \mathrm{~B}$ ). $\mathrm{CD} 13^{+}$and $\mathrm{CD} 44^{+}$cells predominated in mixed-IHCCA as compared to mucin-IHCCA or mucin-pCCA $(P<0.01)$, whereas the opposite was found for $\mathrm{CD} 90^{+}$cells (Figure 3B). In evaluation of marker coexpression, $\mathrm{CD}_{13}{ }^{+} /$ $\mathrm{CD}_{4} 4^{+}$cells predominated (Figure 3C), being more expressed $(P<0.01)$ in mixed-IHCCA $(54 \%)$ than in mucin-IHCCA $(30 \%)$ or mucin-pCCA $(24 \%)$. This was followed by $\mathrm{CD} 90^{+} / \mathrm{CD} 44^{-} / \mathrm{CD} 13^{-}$that predominated in mucin-IHCCAs $(42 \%)$ or mucin-pCCAs $(32 \%)$ versus mixed-IHCCA $(16 \%, P<0.05)$ (Figure 3D). Coexpression of $\mathrm{CD} 90$ with $\mathrm{CD} 13$ or $\mathrm{CD} 44\left(\mathrm{CD} 90^{+} / \mathrm{CD} 44^{+}, \mathrm{CD} 90^{+} /\right.$ $\left.\mathrm{CD}^{+} 3^{+}\right)$occurs rarely $(<10 \%$ cells $)$.

By real-time PCR, vimentin mRNA showed the highest level of expression in cultures of human CCAs (20 to 30 passages) (Supplemental Figure S3), whereas EpCAM and LGR5 were minimally expressed. When CCA subtypes were compared, corroborating data from $\mathrm{FC}, \mathrm{CD} 44$, and CD13 mRNA were more expressed $(P<0.01)$ in mixedCCAs than mucin-CCAs (both IHCCA and pCCA). By contrast, $\mathrm{CD} 90$ and $\mathrm{CD} 133$ were more expressed in mucinCCAs (both IHCCA and pCCA; $P<0.05$ ) than in mixedIHCCAs (Supplemental Figure S3). Finally, the marker of intestinal $^{21}$ and hepatic ${ }^{22}$ stem cells, LGR5, although expressed at very low levels (Supplemental Figure S3), was higher in mucin-IHCCA or mucin-pCCA than mixedIHCCAs $(P<0.05)$.

\section{In Vitro Tumorigenicity}

\section{Spheroid Formation}

The number of spheroids formed in vitro from a given number of seeded cells is a self-renewal index. ${ }^{23}$ We determined the capacity of spheroid formation by cell subpopulations immunosorted from CCA primary cultures (20 to 30 passages). Each subpopulation formed spheroids efficiently, reaching a size of 100 to $500 \mu \mathrm{m}$ after 7 days in culture (Figure 4A). IF (Figure 4A) demonstrated the relative purity of spheroids that were enriched for cells expressing a marker used for immunoselection, ie, spheroids formed by CD90 ${ }^{+}$cells were enriched for CD90 ${ }^{+}$cells. By contrast, in spheroids formed by the negative counterpart, the tested subpopulation represented less than $5 \%$ of the cells (ie, in spheroids formed by $\mathrm{CD} 90^{-}$cells, $\mathrm{CD} 90^{+}$cells were $<5 \%$ by IF), indicating that these spheroids are formed by other CSC subpopulations.

By real-time PCR (Supplemental Figure S4), vimentin mRNA showed the highest level of expression in spheroids, whereas E-cadherin was minimally expressed and almost exclusively in spheroids established from cells immunosorted for CD133 and LGR5 and prepared from mucin-IHCCAs $(P<0.05$ versus mixed) (Supplemental Figure S4). The high expression of vimentin, also in spheroids prepared from CSCs expressing classic epithelial markers, such as LGR5, CD133, and EpCAM, is suggestive for EMT, and this is supported by the positive staining for SNAIL and Twist (Figure 4B). In 

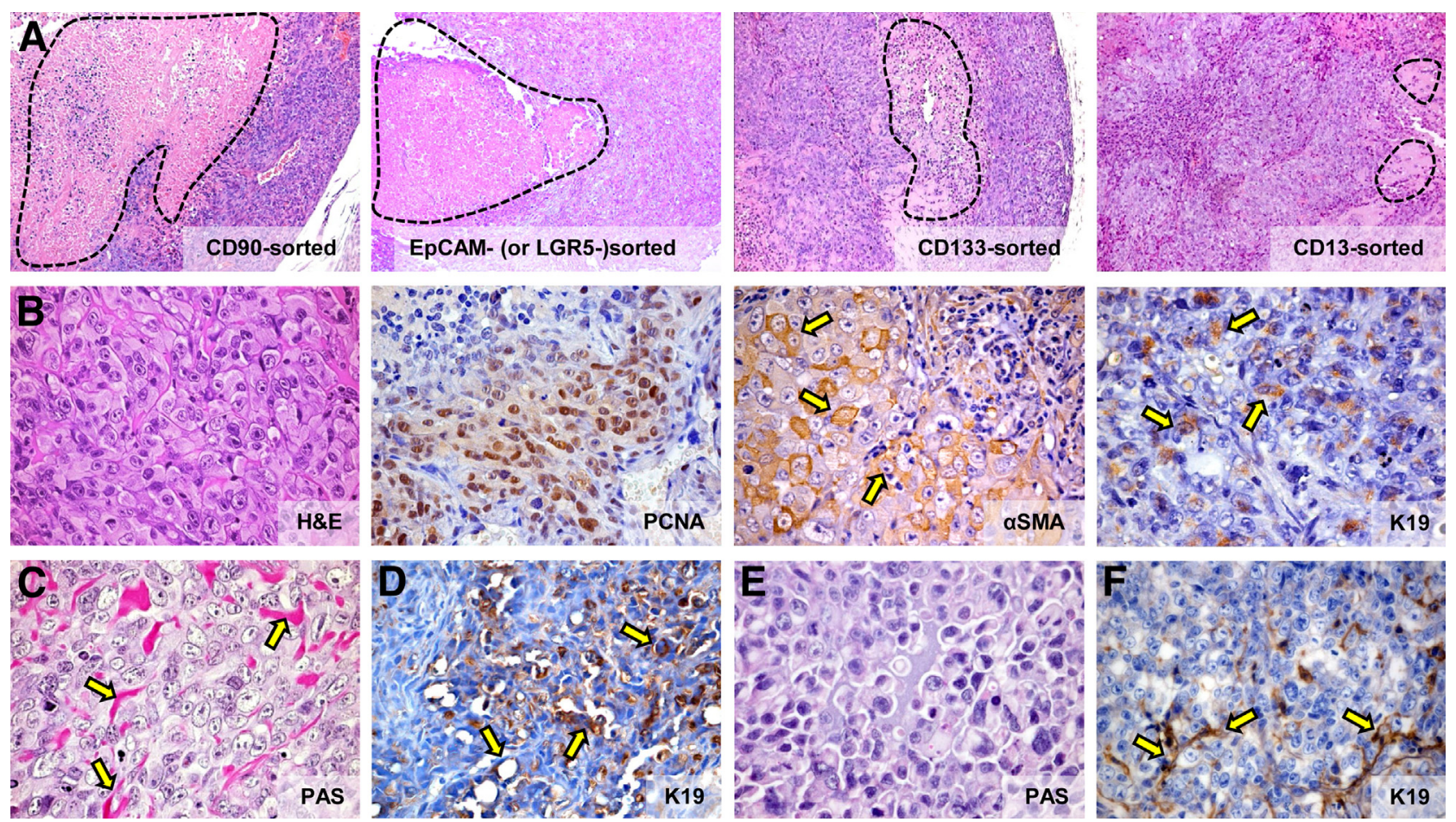

Figure 5 Histology of s.c. human tumor xenografts. A: Hematoxylin and eosin (H\&E): Xenografts arising from CD90 ${ }^{+}$, EpCAM ${ }^{+}$, and LGR5 $5^{+}$spheroids are characterized by the presence of larger necrotic areas in comparison with tumors obtained from $\mathrm{CD}_{133^{+}}$cells, whereas in xenografts from $\mathrm{CD} 13^{+}$spheroids, only few and restricted necrotic areas are observed. Necrotic areas are encircled by the dotted line. B: H\&E: All s.c. xenografts comprise of nests of pleomorphic cells with giant and irregular nuclei and multiple prominent nucleoli. Numerous tumor cells are PCNA positive. Most tumor cells are $\alpha$-SMA positive (arrows), whereas few and scattered nests of tumor cells express K19 (arrows). C: Spheroids from mucin-IHCCA, injected s.c., give rise to xenografts in which neoplastic cells are surrounded by a Periodic-acid Schiff (PAS)-positive material (arrows). D: $\mathrm{EpCAM}^{+}$and $\mathrm{LGR5}^{+}$spheroids from mucin-IHCCA show the presence of few areas composed of $\mathrm{K}_{1} \mathrm{9}^{+}$duct-like structures (arrows). E: Spheroids from mixed-IHCCA give rise to xenografts that are almost PAS negative. F: $5 \%$ to $30 \%$ of cells are K19 positive and form few ductular-like structures (arrows). Original magnification: $\times 10($ A); $\times 40($ B - F).

addition, all spheroids showed a significant expression of markers of pluripotency (OCT4, SOX2) and self-renewal (SALL-4) (Supplemental Figure S4). Interestingly, all spheroids showed a high P-cadherin/E-cadherin ratio (Supplemental Figure S4) that characterizes neoplastic cells with high cancerogenic potential. ${ }^{24}$ As for primary cultures, spheroids cells were negative for CD163, GFAP, CD31, FAP, and SDF1 (not shown).

The number of spheroids was significantly higher (Figure 4A) $(P<0.05)$ for the immunoselected subpopulation in comparison with the negative counterpart. In general, subpopulations of cells immunosorted for epithelial CSC markers (CD133, EpCAM, LGR5) formed a higher $(P<0.01)$ (Figure 4A) number of spheroids as compared to cells selected for $\mathrm{CD} 90$ or for CD13. By comparing mixed- versus mucinIHCCAs, $\mathrm{CD}_{13}{ }^{+}$cells immunoselected from mixed-IHCCAs formed a higher $(P<0.05)$ number of spheroids than $\mathrm{CD} 13^{+}$ cells immunoselected from mucin-IHCCAs, whereas the opposite was found for $\mathrm{CD} 90^{+}$or $\mathrm{CD} 133^{+}$cells, that formed more spheroids when immunoselected from mucin-IHCCA (Figure 4A).

\section{S.C. Tumor Xenografts}

Spheroids (approximately 10,000 cells) were injected s.c. in male NOD/SCID mice, and the volume and number of tumor xenografts were evaluated after 2 months (Table 4). Spheroids dominated by $\mathrm{CD} 90^{+}$or $\mathrm{CD}_{13}{ }^{+}$cells formed tumors in almost all animals (Table 4) and with the highest volume, independently of whether they were from primary cultures of mucin- or mixed-CCAs. Spheroids constituted by CSCs immunosorted for epithelial markers (EpCAM, LGR5, CD133), yielded tumors less frequently and mainly when $\mathrm{CD}_{133^{+}}$spheroids were implanted (Table 4). LGR5 ${ }^{+}$ and $\mathrm{EpCAM}^{+}$spheroids formed tumors only after 3 to 4 months.

Tumor xenografts were characterized by necrotic areas (Figure 5A and Table 5). In particular, xenografts arising from $\mathrm{CD} 0^{+}, \mathrm{EpCAM}^{+}$, and $\mathrm{LGR}^{+}$spheroids showed the presence of larger necrotic areas $(51.16 \% \pm 5.13 \%$, $44.24 \% \pm 7.18 \%, 47.85 \% \pm 5.77 \%$, respectively) in comparison with tumors obtained from $\mathrm{CD}_{13} 3^{+}$spheroids $(29.84 \% \pm 6.45 \%, P<0.05)$. Xenografts from $\mathrm{CD} 13^{+}$cells contained only a few, small necrotic areas $(19.84 \% \pm 4.59 \%$, $P<0.05$ ) (Figure 5A). Morphologically, all s.c. xenografts consisted of nests of pleomorphic cells with giant and irregular nuclei and multiple prominent nucleoli (Figure 5B). Tumor cells highly expressed markers of proliferation such as PCNA (Figure 5B) and were positive for stromal markers such as desmin, vimentin, and $\alpha$-SMA (Figure 5B). Some nests of tumor cells expressed CK19 (Figure 5B). 
Table 5 Summary of Morphological and IHC Features of S.C. Xenografts*

\begin{tabular}{|c|c|c|c|c|c|c|c|c|c|c|}
\hline \multirow[b]{3}{*}{ Necrosis } & \multicolumn{5}{|c|}{ Xenographs from mucin-IHCCA } & \multicolumn{5}{|c|}{$\underline{\text { Xenographs from mixed-IHCCA }}$} \\
\hline & CD90 & EpCAM & LGR5 & CD133 & CD13 & CD90 & ЕрСАM & LGR5 & CD133 & CD13 \\
\hline & \multicolumn{3}{|c|}{ Large areas } & Small areas & Focal & \multicolumn{3}{|c|}{ Large areas } & Small areas & Focal \\
\hline \multicolumn{11}{|c|}{ Histopathological aspects mimicking human CCA features } \\
\hline PAS positivity & - & + & + & + & + & - & - & - & - & - \\
\hline
\end{tabular}

*The table summarizes morphological and IHC features of s.c. xenografts in Figure 5.

$\mathrm{BD}$, bile duct; CCA, cholangiocarcinoma; IHC, immunohistochemistry; IHCCA, intrahepatic cholangiocarcinoma.

Spheroids prepared from mucin-IHCCAs, including $\mathrm{EpCAM}^{+}, \mathrm{LGR}^{+}, \mathrm{CD} 133^{+}$, and $\mathrm{CD}_{13}{ }^{+}$spheroids, promoted tumor xenografts composed of cells surrounded by a PAS-positive material (Figure 5C). In xenografts from $\mathrm{EpCAM}^{+}$and $\mathrm{LGR}^{+}$spheroids, $\mathrm{K} 9^{+}$duct-like structures were focally present (Figure 5D), mimicking the histomorphological aspect of the tumor of origin (ie, mucinIHCCAs), this being absent with $\mathrm{CD} 133^{+}$and $\mathrm{CD} 13^{+}$ spheroids. Finally, xenografts from $\mathrm{CD} 90^{+}$spheroids were PAS and K19 negative (Table 5). No differences were observed between tumor xenografts obtained from mucinIHCCAs or mucin-pCCAs.

In all xenografts obtained by the injection of spheroids prepared from mixed-IHCCA cultures, no PAS positivity was observed (Figure 5E); few areas, representing 5\% to 30\% of the tumor mass, were formed by cholangiolo-like (ductular) structures (K19) (Figure 5F), recalling the histopathological aspects of the tumor of origin (ie, mixed-IHCCA).

\section{Intrahepatic Tumor Xenografts}

The spheroids that most effectively formed s.c. tumor xenografts (ie, $\mathrm{CD} 133^{+}, \mathrm{CD} 13^{+}$, and $\mathrm{CD} 90^{+}$) were injected (approximately 10,000 cells) directly into the liver of normal and cirrhotic (carbon tetrachloride-induced) NOD/SCID mice. No tumor was observed 1 month after the injection of spheroids in the livers of normal mice $(n=3$ each for $\mathrm{CD} 3^{+}, \mathrm{CD} 133^{+}$, and $\mathrm{CD} 90^{+}$spheroids from mucin- or mixed-IHCCA). By contrast, in cirrhotic mice injected $(n=18)$ with spheroids prepared from cultures of mixed- or mucin-IHCCA, several tumor masses were recognizable (Figure 6A), with number and volume being much higher for $\mathrm{CD}_{13}{ }^{+}$and $\mathrm{CD} 90^{+}$than $\mathrm{CD} 133^{+}$spheroids $(P<0.01)$.

Morphologically, the epithelial components predominated over the stromal components, the positivity for stromal markers being restricted to fewer tumor cells in comparison with s.c. xenografts (Figure 6). Tumors (Figure 6B) were composed of cords of polygonal cells with irregular nuclei and prominent nucleoli (Figure 6, B and C) with vascular invasion being present in all specimens (Figure 6B). At the center of tumor masses, nearly $10 \%$ of cells expressed stromal markers such as $\alpha$-SMA (Figure 6D), whereas at the periphery and close to liver parenchyma, tumor cells presented a more differentiated phenotype characterized by the presence of duct-like structures (Figure $6 \mathrm{E}$ ), $\mathrm{PAS}^{+}$cells (Figure $6 \mathrm{~F}$ ), cords of $\mathrm{HepPar}-\mathrm{1}^{+}$cells (Figure $6 \mathrm{G}$ ), and $\mathrm{K} 19^{+}$ductular structures (Figure $6 \mathrm{H}$ ). No morphological difference was found among tumors generated by $\mathrm{CD} 133^{+}, \mathrm{CD} 90^{+}$, and $\mathrm{CD} 13^{+}$spheroids. By contrast, PAS positivity was mainly present in tumors derived from spheroids isolated from mucin-IHCCAs, whereas HepPar- $1^{+}$cells were only present in tumors derived from mixed-IHCCAs. These pathological features reassembled the phenotype of the original human CCAs (Figure 2A).

In additional experiments performed in immunocompetent cirrhotic BALB/c mice, under pharmacological immunosuppression, $\mathrm{CD} 133^{+}$spheroids prepared from mucinIHCCA $(n=4)$ reproduced a tumor almost totally

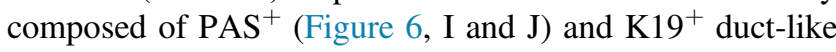
structures, with pathological features very similar to human mucin-IHCCAs (Figure 2A).

\section{Discussion}

According to a recent classification, ${ }^{2}$ CCAs are comprised of a pure mucin-secreting form located intrahepatically or at the hilum, and a mixed form with a peripheral location in the liver. This distinction reflects the cells of origin because pathology and molecular analyses ${ }^{2}$ indicate that mucinCCAs derive from the mucin-secreting epithelium lining large ducts or from PBGs. By contrast, the mixed form derives from cuboidal non-mucin-secreting cells lining bile ductules or canals of Hering. The analyses performed in our study further support this classification because mucinCCAs showed a similar profile in terms of CSC subpopulations, regardless of whether intrahepatic or perihilar, whereas there were significant differences found between mucin- and mixed-IHCCAs.

We firstly analyzed established cell lines that matched the recent CCA classification. ${ }^{2}$ We observed how those originating from pCCA or gallbladder cancer were mucin positive, whereas those derived from human IHCCA were either mucin positive or negative; the profile of CSC markers was similar among mucin-positive cell lines regardless of their origin from IHCCA, pCCA, or gallbladder cancer, but were 

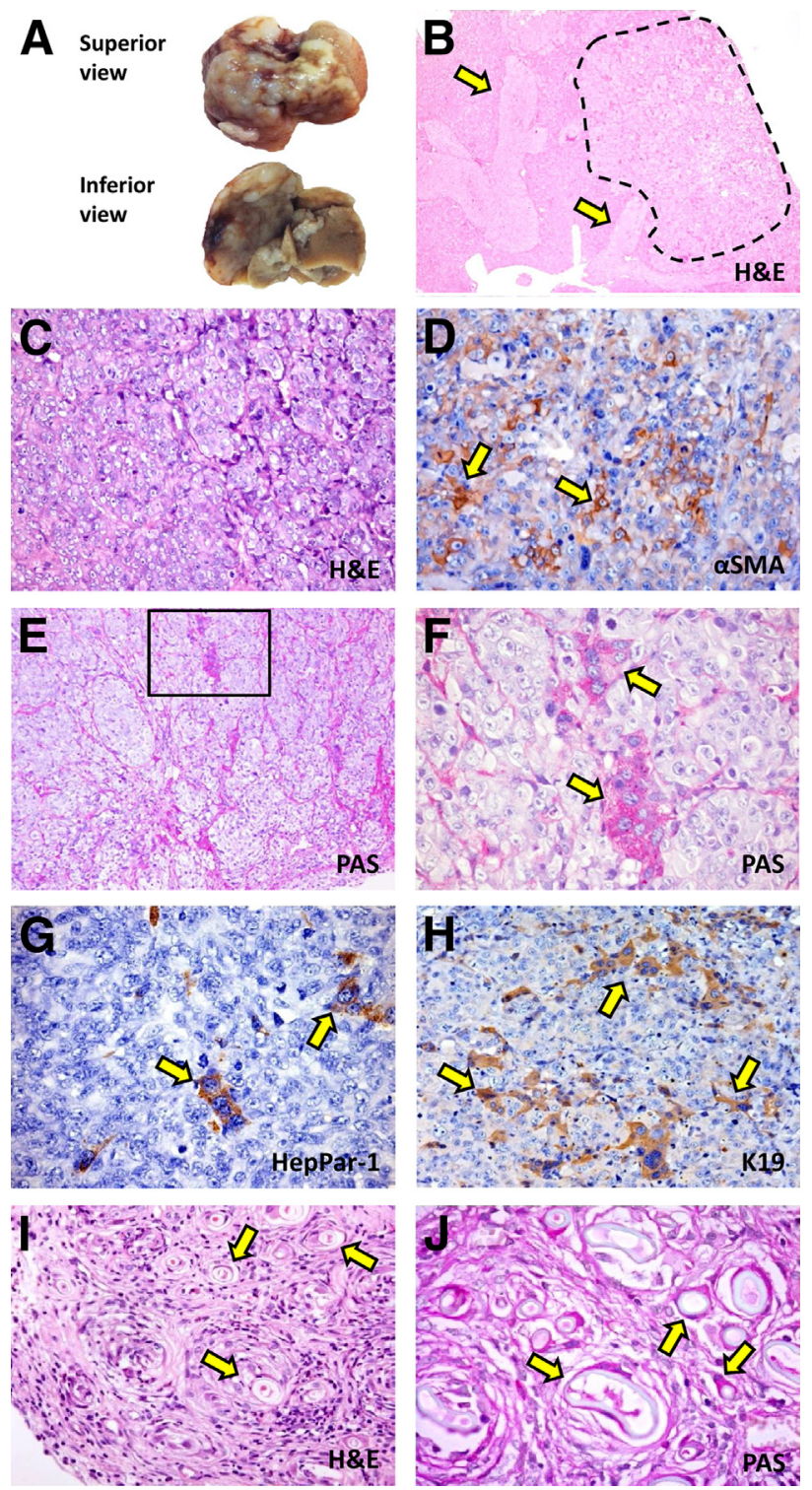

Figure 6 Intrahepatic tumor xenografts: morphological and phenotypic features. Injection of spheroids (approximately 10,000 cells) formed by cells immunoselected for a determined cholangiocarcinoma (CCA) marker into the livers of cirrhotic SCID mice (carbon tetrachloride-induced) leads, after 4 weeks, to evident liver cancers. A: Tumor masses observed 4 weeks after intrahepatic injection of $\mathrm{CD}^{+} 0^{+}$spheroids from primary cultures of mucin-IHCCA. B: Hematoxylin and eosin (H\&E): The liver is occupied by several tumor masses (dotted line); vascular invasion (arrows). C: H\&E: Tumor masses were composed of strands of polygonal cells with giant nuclei and prominent nucleoli. D: Immunohistochemistry (IHC): At the center of tumor masses, $\alpha$-SMA-positive tumor cells are present (arrows). E-H: At the periphery of the tumor masses, Periodic-acid Schiff (PAS)positive cells (F, arrows), cords of HepPar-1-positive cells (G, arrows), and $\mathrm{K} 19$ ductular-like structures (H, arrows) are present, reproducing a moderately differentiated carcinoma. $\mathbf{E}=$ PAS staining: Area in the box is magnified in $\mathbf{F} ; \mathbf{F}=$ PAS staining: $\mathbf{G}=$ IHC for HepPar-1. $\mathbf{H}=$ IHC for CK19. I and J: Immunocompetent cirrhotic BALBC mice, under pharmacological immunosuppression, injected with $\mathrm{CD}_{133^{+}}$spheroids prepared from mucin-IHCCA primary cultures. Four weeks after intrahepatic injection, a tumor almost totally composed of $\mathrm{PAS}^{+}$duct-like structures (arrows) is observed. I $=$ H\&E: $\mathbf{J}=$ PAS staining. $n=4$ (I and $\mathbf{J}$, mucin-IHCCA primary cultures). Original magnification: $\times 10(\mathbf{B}$ and $\mathbf{E}) ; \times 20(\mathbf{C}, \mathbf{D}, \mathbf{H}$, and $\mathbf{I}) ; \times 40(\mathbf{F}, \mathbf{G}$, and $\mathbf{J})$. completely different with respect to mucin-negative cell lines. All established cell lines were characterized by a predominance of specific CSCs, suggesting that each cell line derived from a single cell population that was present in the original cancer.

On the basis of the CSC subpopulations identified in established cell lines and on suggestions from recent reports, ${ }^{5,6,8-10,22,25}$ we analyzed (by IHC) a panel of CSC markers in human CCA samples. We demonstrated how the positivity for CSC markers characterizes a high percentage $(>30 \%)$ of CCA cells. This is in sharp contrast with hepatocellular carcinomas ${ }^{3}$ or other solid tumors ${ }^{4}$ where these markers identify only $0.5 \%$ to $3 \%$ of the tumor cells. However, these markers are expressed also by normal stem/ progenitor cells resident in the liver and biliary tree. Specifically, LGR5 and CD133 identify the primitive stem cells at or near the bottoms (the crypts) of PBGs, whereas EpCAM labels the transit/amplifying and intermediate cell compartments of the same glands. ${ }^{18}$ Cells residing in the canals of Hering and bile ductules are also composed in part of $\mathrm{CD}_{133^{+}}$and $\mathrm{EpCAM}^{+}$cells, whereas CD90 and CD13 diffuse positivity characterizes, respectively, stromal (periportal and periglandular) and parenchymal cells. ${ }^{18}$ These compartments are activated and expanded in the course of chronic liver damage ${ }^{26}$ Because most CCAs emerge in the context of chronic inflammation of liver parenchyma or bile ducts, the abundance of CSC markers we have found in human CCAs is not surprising, given the expansion of stem/ progenitor cell populations in this inflammatory background. ${ }^{1,25,26}$ Consistently, a recent study demonstrated that most cells in human CCAs coexpress K19 and albumin, ${ }^{10}$ a feature characterizing the transit/amplifying compartment of PBGs and stem/progenitors in ductular reactions. ${ }^{18}$ On the other hand, apart from CD13, CSC markers are not expressed in normal mature cholangiocytes lining bile ducts, ${ }^{18}$ and this supports the interpretation of origin of CCAs from stem/progenitors rather than fully differentiated cells. Recently, consistent with our conclusions, a key role for hypermethylation of multiple $\mathrm{CpG}$ sites at genes associated with a stem cell-like phenotype has been shown as a common molecular aberration in CCA. ${ }^{27}$

In general, whereas all CCA cases showed a diffuse positivity for CD44, CD90, EpCAM, and CD133, CD13 positivity identified mixed CCAs. This was also confirmed in primary cultures where $\mathrm{CD} 13^{+}$cells were mainly of the $\mathrm{CD} 13^{+} / \mathrm{CD} 44^{+}$phenotype. CD13, a marker of quiescent CSCs in different cancers, ${ }^{3,4}$ confers resistance to chemoradio therapies and is responsible for tumor recurrence, where CD13 can give rise to different progeny, including $\mathrm{CD}^{+} 0^{+}$cells. ${ }^{3,4,8}$ In human CCA, the expression of CD44 (a receptor for hyaluronic acid) showed an association with periductal infiltrative type, poor differentiation, and vascular invasion. ${ }^{7}$ By contrast, LGR5 was found to identify mucinCCAs as demonstrated in situ by IHC. Unfortunately, epithelial cells did not survive well in primary cultures, and therefore, correspondence between IHC in situ and FC in 
primary cultures could not be evaluated. Nevertheless, realtime PCR confirmed the higher expression of this marker in mucin-IHCCA than mixed-IHCCA primary cultures. LGR5 marks stem cells in multiple adult organs ${ }^{18,21,22}$ and CSCs in colorectal ${ }^{21}$ and ductal pancreatic adenocarcinoma. ${ }^{28}$ In the liver and biliary tree, LGR5 identifies a subset of multipotent stem cells in PBGs. ${ }^{18}$ This supports our hypothe$\mathrm{sis}^{25}$ that pure mucin-CCAs represent cancers originating from PBGs, closely related biologically to intestinal and pancreatic cancers. Indeed, LGR5 ${ }^{+}$cells in glandular crypts or in pancreatic duct glands play a role in oncogenic processes. $^{12,28}$ Consistently, K-ras mutations, frequent in cancers of colon and pancreas, ${ }^{21,28}$ have been observed only in mucin- but not mixed-CCAs. ${ }^{2}$ Therefore, the similarities between colorectal, ductal pancreatic cancers and mucinCCAs suggest an origin from similar glands and endodermal stem/progenitors. ${ }^{25}$ It is noteworthy that almost all CCA cells are positive for nestin. Nestin is a class VI intermediate filament protein, which has received large attention as a cancer stem cell marker in various tumors, including gastrointestinal cancers. ${ }^{29}$

In situ, $30 \%$ of CCA cells express EMT markers with a cell subpopulation coexpressing mesenchymal and epithelial markers. The expression of EMT markers in human extrahepatic CCA has been recently considered a significant prognostic factor with negative impact on survival. ${ }^{30}$ When primary cultures were prepared from human CCA samples, the mesenchymal cell components largely dominated with significant expression of EMT markers and loss of E-cadherin, even though these cultures were almost negative for markers of tumor-associated macrophages, hepatic stellate cells, endothelial cells, and fibroblasts. This could be caused by the culture selection for the mesenchymal cells or the subpopulation of epithelial cells expressing EMT traits and the induction of EMT by our culture conditions. However, the CSC profiles were similar between mucin-IHCCA or mucin-pCCA in primary cultures also. By contrast, $\mathrm{CD} 13^{+}$ and $\mathrm{CD}_{4} 4^{+}$cells predominated in mixed-IHCCAs as compared to mucin-IHCCAs or mucin-pCCAs; the opposite was observed for $\mathrm{CD} 90^{+}$cells.

Depending on the microenvironment, the behavior of CSC subpopulations largely differed. In vitro, for example, epithelial CSCs (CD133, EpCAM, and LGR5) showed the highest tumorigenic potential in terms of both number and size of spheroids whereas different findings were obtained in vivo (tumor xenografts), where $\mathrm{CD} 90^{+}$and $\mathrm{CD} 13^{+}$cells were more tumorigenic. Because spheroids were highly enriched for the immunosorted CSC subpopulation, we used spheroids for s.c. or intrahepatic xenografts. Unfortunately, the mass of available surgical pieces is very frequently inadequate for CSC isolation and direct xenografting. Spheroids were mostly formed by cells with EMT traits (SNAIL, Twist, negative E-cadherin) and expressing markers of pluripotency (Nanog, OCT4, Sox2) and selfrenewal (SALL-4); this was found also in spheroids of CSCs expressing CD133, EpCAM, and LGR5. For primary cultures also, spheroids were almost negative for markers of tumor-associated macrophages, hepatic stellate cells, endothelial cells, and fibroblasts. In all s.c. tumor xenografts, tumors were mostly composed of anaplastic cells mimicking stromal tumors. Interestingly, in s.c. xenografts, few $\mathrm{K} 19^{+} /$ PAS ductular structures were observed when CSCs were selected from mixed-IHCCA, whereas $\mathrm{PAS}^{+}$cells were present when CSCs were selected from mucin-IHCCA, thus reproducing some features of the original carcinomas.

Cancers with features of carcinomas were reproduced after injection of spheroids in cirrhotic livers, further confirming the role of the microenvironment in determining the cancer phenotype. The cirrhotic liver evidently facilitates CSC engraftment and growth, and this is consistent with the frequent emergence of IHCCAs in the context of cirrhosis. ${ }^{1,25}$ Remarkably, we were able to reproduce several morphological and IHC features of human CCA. Pas-positive areas, typical of mucin-IHCCA, for example, were reproduced with spheroids prepared from mucin-IHCCA cultures, whereas Hep-Par- $1^{+}$areas, typical of mixed-CCA, were reproduced with spheroids from mixed-IHCCA cultures. Interestingly, when $\mathrm{CD} 133^{+}$spheroids from mucin-IHCCA were injected in immunocompetent mice, a tumor very similar to the original human mucin-IHCCA was reproduced. CD133, expressed by angioblasts ${ }^{31}$ as well as by endodermal stem/ progenitors, ${ }^{32}$ marks the stem cell compartment at the bottom of $\mathrm{PBGs}^{18}$ and identifies CSC niches in different solid carcinomas, ${ }^{33}$ including $\mathrm{HCC}^{34}$ and CCA. ${ }^{1}$

In general, the changing phenotype of s.c. versus intrahepatic xenografts indicates the plasticity of CCA CSCs and/or the impact of the environment on selection of the mesenchymal versus epithelial components in the spheroids or by favoring EMT.

In conclusion, our study clearly demonstrated that CSCs are heterogeneously and largely represented in human CCAs, implicating these cancers as diseases rich in stem/progenitor cells. A consistent subpopulation of CSCs expresses the EMT trait and showed marked biological plasticity. The normal counterparts to these CSCs are present in the normal biliary tree, suggesting that proliferation, expansion, and neoplastic transformation of these cells could represent the basis for the emergence of human CCAs, the phenotype of CCA depending on the site of origin, the plasticity of the CSCs, and the microenvironment.

\section{Acknowledgments}

We thank Dr. Anthony J. Demetris (University of Pittsburgh, Pittsburgh, PA) for CCLP-1 and HUCCT-1 cells, the Cancer Cell Repository (Tohoku University, Sendai, Japan) for HuH-28 cells, Dr. Yoshiyuki Ueno (the Cancer Cell Repository, Tohoku University, Sendai, Japan) for TFK-1 cells, and Dr. J. Gregory Fitz (University of Texas Southwestern, Dallas, TX) for Mz-ChA1 cells. 


\section{Supplemental Data}

Supplemental material for this article can be found at http://dx.doi.org/10.1016/j.ajpath.2015.02.010.

\section{References}

1. Rizvi S, Gores GJ: Pathogenesis, diagnosis, and management of cholangiocarcinoma. Gastroenterology 2013, 145:1215-1229

2. Komuta M, Govaere O, Vandecaveye V, Akiba J, Van Steenbergen W, Verslype C, Laleman W, Pirenne J, Aerts R, Yano H, Nevens F, Topal B, Roskams T: Histological diversity in cholangiocellular carcinoma reflects the different cholangiocyte phenotypes. Hepatology 2012, 55:1876-1888

3. Yamashita T, Wang XW: Cancer stem cells in the development of liver cancer. J Clin Invest 2013, 123:1911-1918

4. Magee JA, Piskounova E, Morrison SJ: Cancer stem cells: impact, heterogeneity, and uncertainty. Cancer Cell 2012, 21:283-296

5. Leelawat K, Thongtawee $\mathrm{T}$, Narong $\mathrm{S}$, Subwongcharoen $\mathrm{S}$, Treepongkaruna SA: Strong expression of CD133 is associated with increased cholangiocarcinoma progression. World J Gastroenterol 2011, 17:1192-1198

6. Sulpice L, Rayar M, Turlin B, Boucher E, Bellaud P, Desille M, Meunier B, Clément B, Boudjema K, Coulouarn C: Epithelial cell adhesion molecule is a prognosis marker for intrahepatic cholangiocarcinoma. J Surg Res 2014, 192:117-123

7. Gu MJ, Jang BI: Clinicopathologic significance of Sox2, CD44 and CD44v6 expression in intrahepatic cholangiocarcinoma. Pathol Oncol Res 2014, 20:655-660

8. Haraguchi N, Ishii H, Mimori K, Tanaka F, Ohkuma M, Kim HM, kita H, Takiuchi D, Hatano H, Nagano H, Barnard GF, Doki Y, Mori M: CD13 is a therapeutic target in human liver cancer stem cells. J Clin Invest 2010, 120:3326-3339

9. Sukowati CH, Anfuso B, Torre G, Francalanci P, Crocè LS, Tiribelli C: The expression of CD90/Thy-1 in hepatocellular carcinoma: an in vivo and in vitro study. PLoS One 2013, 8:e76830

10. Thanan R, Pairojkul C, Pinlaor S, Khuntikeo N, Wongkham C, Sripa B, Ma N, Vaeteewoottacharn K, Furukawa A, Kobayashi H, Hiraku Y, Oikawa S, Kawanishi S, Yongvanit P, Murata M: Inflammation-related DNA damage and expression of CD133 and Oct3/4 in cholangiocarcinoma patients with poor prognosis. Free Radic Biol Med 2013, 65:1464-1472

11. Carpino G, Cardinale V, Onori P, Franchitto A, Berloco PB, Rossi M, Wang Y, Semeraro R, Anceschi M, Brunelli R, Alvaro D, Reid LM, Gaudio E: Biliary tree stem/progenitor cells in glands of extrahepatic and intrahepatic bile ducts: an anatomical in situ study yielding evidence of maturational lineages. J Anat 2012, 220:186-199

12. Chomczynski P, Sacchi N: Single-step method of RNA isolation by acid guanidinium thiocyanate-phenol-chloroform extraction. Anal Biochem 1987, 162:156-159

13. Turner RA, Mendel G, Wauthier E, Barbier C, Reid LM: Hyaluronansupplemented buffers preserve adhesion mechanisms facilitating cryopreservation of human hepatic stem/progenitor cells. Cell Transplant 2012, 21:2257-2266

14. Walker F, Zhang HH, Odorizzi A, Burgess AW: LGR5 is a negative regulator of tumourigenicity, antagonizes Wnt signalling and regulates cell adhesion in colorectal cancer cell lines. PLoS One 2011, 6:e22733

15. Oikawa T, Kamiya A, Zeniya M, Chikada H, Hyuck AD, Yamazaki Y, Wauthier E, Tajiri H, Miller LD, Wang XW, Reid LM, Nakauchi H: Sal-like protein 4 (SALL4), a stem cell biomarker in liver cancers. Hepatology 2013, 57:1469-1483
16. Li H, Richard K, McKinnon B, Mortimer RH: Effect of iodide on human choriogonadotropin, sodium-iodide symporter expression, and iodide uptake in BeWo choriocarcinoma cells. J Clin Endocrinol Metab 2007, 92:4046-4051

17. D'Argenio G, Amoruso DC, Mazzone G, Vitaglione P, Romano A, Ribecco MT, D'Armiento MR, Mezza E, Morisco F, Fogliano V, Caporaso N: Garlic extract prevents $\mathrm{CCl}(4)$-induced liver fibrosis in rats: the role of tissue transglutaminase. Dig Liver Dis 2010, 42:571-577

18. Cardinale V, Wang Y, Carpino G, Mendel G, Alpini G, Gaudio E, Reid LM, Alvaro D: The biliary tree-a reservoir of multipotent cells. Nat Rev Gastroenterol Hepatol 2012, 9:231-240

19. Sirica A: The role of cancer-associated myofibroblasts in intrahepatic cholangiocarcinoma. Nat Rev Gastroenterol Hepatol 2011, 9:44-54

20. Joyce JA, Pollard JW: Microenvironmental regulation of metastasis. Nat Rev Cancer 2009, 9:239-252

21. Zeki SS, Graham TA, Wright NA: Stem cells and their implications for colorectal cancer. Nat Rev Gastroenterol Hepatol 2011, 8:90-100

22. Huch M, Dorrell C, Boj SF, van Es JH, Li VS, van de Wetering M, Sato T, Hamer K, Sasaki N, Finegold MJ, Haft A, Vries RG, Grompe M, Clevers H: In vitro expansion of single Lgr5+ liver stem cells induced by Wnt-driven regeneration. Nature 2013, 494:247-250

23. Fang D, Nguyen TK, Leishear K, Finko R, Kulp AN, Hotz S, an Belle PA, Xu X, Elder DE, Herlyn M: A tumorigenic subpopulation with stem cell properties in melanomas. Cancer Res 2005, 65: 9328-9337

24. Patel IS, Madan P, Getsios S, Bertrand MA, MacCalman CD: Cadherin switching in ovarian cancer progression. Int J Cancer 2003, 106: $172-177$

25. Cardinale V, Carpino G, Reid L, Gaudio E, Alvaro D: Multiple cells of origin in cholangiocarcinoma underlie biological, epidemiological and clinical heterogeneity. World J Gastrointest Oncol 2012, 4:94-102

26. Alvaro D, Mancino MG, Glaser S, Gaudio E, Marzioni M, Francis H, Alpini G: Proliferating cholangiocytes: a neuroendocrine compartment in the diseased liver. Gastroenterology 2007, 132:415-431

27. Sriraksa R, Zeller C, Dai W, Siddiq A, Walley AJ, Limpaiboon T, Brown R: Aberrant DNA methylation at genes associated with a stem cell-like phenotype in cholangiocarcinoma tumors. Cancer Prev Res (Phila) 2013, 6:1348-1355

28. Amsterdam A, Raanan C, Schreiber L, Polin N, Givol D: LGR5 and Nanog identify stem cell signature of pancreas beta cells which initiate pancreatic cancer. Biochem Biophys Res Commun 2013, 433:157-162

29. Ishiwata T, Matsuda Y, Naito Z: Nestin in gastrointestinal and other cancers: effects on cells and tumor angiogenesis. World J Gastroenterol 2011, 17:409-418

30. Nitta $T$, Mitsuhashi $T$, Hatanaka $Y$, Miyamoto $M$, Oba $K$, Tsuchikawa T, Suzuki Y, Hatanaka KC, Hirano S, Matsuno Y: Prognostic significance of epithelial-mesenchymal transition-related markers in extrahepatic cholangiocarcinoma: comprehensive immunohistochemical study using a tissue microarray. Br J Cancer 2014, 111:1363-1372

31. Hristov M, Weber C: Endothelial progenitor cells: characterization, pathophysiology, and possible clinical relevance. J Cell Mol Med 2004, 8:498-508

32. Schmelzer E, Zhang L, Bruce A, Wauthier E, Ludlow J, Yao HL, Moss N, Melhem A, McClelland R, Turner W, Kulik M, Sherwood S, Tallheden T, Cheng N, Furth ME, Reid LM: Human hepatic stem cells from fetal and postnatal donors. J Exp Med 2007, 204:1973-1987

33. Mizrak D, Brittan M, Alison MR: CD133: molecule of the moment. J Pathol 2008, 214:3-9

34. Song W, Li H, Tao K, Li R, Song Z, Zhao Q, Zhang F, Dou K: Expression and clinical significance of the stem cell marker CD133 in hepatocellular carcinoma. Int J Clin Pract 2008, 62:1212-1218 